\title{
2-苯甲酰基嘧啶类化合物的合成与生物活性
}

\author{
吕强 $a, b$ 芦昕婷 $b$ 戴 明 $b$ 刘世梦 $b$ \\ 朱为宏 ${ }^{a}$ 杜苑 $*, c$ 吕龙 ${ }^{b}$ \\ ( ${ }^{a}$ 华东理工大学化学与分子工程学院 上海 200237) \\ ( ${ }^{b}$ 中国科学院上海有机化学研究所 有机氟化学重点实验室 上海 200032) \\ $\left({ }^{c}\right.$ 上海应用技术学院化学与环境工程学院 上海 201418)
}

\begin{abstract}
摘要 2 -嘧啶氧基- $N$-芳基茮胺类化合物结构经过两次骨架结构优化后得到 2-苯甲酰基嘧啶类化合物二次先导结构. 在 二次先导结构基础上, 共设计并合成了 36 个化合物, 所有化合物结构经 ${ }^{1} \mathrm{H} N \mathrm{NM} 、{ }^{13} \mathrm{C} \mathrm{NMR} 、 \mathrm{HRMS}$ 确认, 并进行了 室内杀菌活性篮选，对各部位取代基进行了逐次优化. 结果表明 2-苯甲酰基嘧啶类化合物中 $\mathrm{R}^{1}$ 取代基以 2 位卤素或烷 基取代的苯环或杂环活性最好; 中间苯环 6 位引入氟原子活性保持; 嘧啶环 4,6 位甲氧基取代活性较好, 5 位甲基取代 活性大大降低; 羊基被还原为羟基后活性消失. 其中 2,3-二氯- $N$-[2-(4,6-二甲氧基嘧啶-2-甲酰基)苯氧基]- $N$-甲基苯甲酰 胺(4AHI)、2,5-二氯- $N$-[2-(4,6-二甲氧基嘧啶-2-甲酰基)苯氧基]- $N$-甲基苯甲酰胺(4AHn)及 $N$-[2-(4,6-二甲氧基嘧啶-2-甲 酰基)-3-氟苯氧基]- $N, 2$-二甲基苯甲酰胺(4AFd)对黄瓜白粉病的杀菌活性与对照样苯菌酮相当.
\end{abstract}

关键词 2-苯甲酰基嘧啶; 合成; 结构优化; 生物活性

\section{Synthesis and Bioactivity of 2-Benzoyl Pyrimidine Derivatives}

\author{
Lü, Qiang ${ }^{a, b}$ \\ Lu, Xinting ${ }^{b}$ \\ Dai, Ming ${ }^{b}$ \\ Liu, Shimeng ${ }^{b}$ \\ Zhu, Weihong ${ }^{a}$ \\ $\mathrm{Du}, \mathrm{Pa}^{*, c}$ \\ Lü, Long ${ }^{b}$
}

( ${ }^{a}$ School of Chemistry and Molecular Engineering, East China University of Science and Technology, Shanghai 200237)

( ${ }^{b}$ Key Laboratory of Organofluorine Chemistry, Shanghai Institute of Organic Chemistry, Chinese Academy of Science,

Shanghai 200032)

( ${ }^{c}$ School of Chemical and Environmental Engineering, Shanghai Institute of Technology, Shanghai 201418)

\begin{abstract}
Benzoyl pyrimidine as a secondary leading structure is developed by twice optimization of 2-pyrimidinoxy- $N$ aryl benzylamine. Based on this structure, thirty six 2-benzoyl pyrimidine derivatives have been designed and synthesized. All compounds are determined by ${ }^{1} \mathrm{H}$ NMR, ${ }^{13} \mathrm{C}$ NMR and HRMS. Their interior fungicidal activities show that $\mathrm{R}^{1}$ substituent prefers phenyl or heterocyclic group with 2-halo or 2-alkyl group. When introducing fluoride to 6-position on benzene ring, the fungicidal activity is maintained at a similar level. 4,6-Dimethoxy group on pyrimidine ring was recognized as a best substituent by far. The activity is seriously decreased when methyl group is fixed on 5-position of pyrimidine. Even the fungicidal activities become completely disappeared when the ketone group is reduced to hydroxyl group. Among them, three compounds 2,3-dichloro- $N$-(2-(4,6-dimethoxypyrimidine-2-carbonyl)phenoxy)- $N$-methylbenzamide (4AHI), 2,5-dichloro- $N$-(2-(4,6-dimethoxypyrimidine-2-carbonyl)phenoxy)- $N$-methylbenzamide (4AHn) and $N$-(2-(4,6-dimethoxypyrimidine-2-carbonyl)-3-fluorophenoxy)- $N$,2-dimethylbenzamide (4AFd) exhibit comparable fungicidal activity against cucumber powdery mildew to the reference metrafenone.
\end{abstract}

Keywords 2-benzoyl pyrimidine; synthesis; structral optimization; biological activity

嘧啶作为药效团或边链在农药结构设计和优化当 中被广泛应用, 在除草剂品种中含嘧啶结构的磺酰脲
类、嘧啶水杨酸类化合物为代表的乙酰乳酸合成酶 (ALS) 抑制剂被广泛研究 ${ }^{[1]}$. 在嘧啶水杨酸类化合物的

\footnotetext{
*E-mail: dupa@sit.edu.cn

Received December 18, 2015; revised February 17, 2015; published online February 18, 2015.

Project supported by the National Key Technologies R\&D Program (No. 2011BAE06B02)

“十二五” 国家科技支撑计划(No. 2011BAE06B02)资助项目.
} 
基础上，我们发现了一类具有全新结构和高效除草活性 的 2-嘧啶氧基- $N$-芳基茮胺类化合物 ${ }^{[2]}$. 在对这类化合 物进一步结构优化过程中, 参考了日本组合化学公司新 型除草剂 Pyrimisulfan 结构 ${ }^{[3]}$, 改变嘧啶环和苯环之间 桥连结构, 将氧桥修饰为羰基. 经过室内生物活性测定 后发现，这类化合物基本丧失除草活性，但是表现出一 定的杀菌活性 ${ }^{[4]}$. 进一步优化茮胺边链为羟酰胺边链, 我们发现了一类具有很高的杀菌活性的全新结构化合 物, 本文以此类结构作为先导, 开展了结构优化工作 (图 1).<smiles>[R]c1ccc(NCc2c([X])cccc2Oc2nc(OC)cc(OC)n2)cc1</smiles>

2-嘧啶氧基- $N$-芳基芐胺<smiles></smiles>

Pyrimisulfan

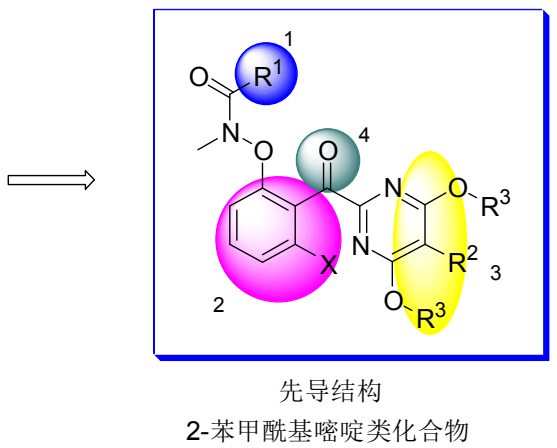

图 1 先导结构及其结构优化

Figure 1 Lead structure and its structural optimization

2-苯甲酰基嘧啶类化合物结构优化工作主要集中 在以下几个方面: (1)首先优化 $\mathrm{R}^{1}$ 取代基; (2)优化苯环 $\mathrm{X}$ 取代基; (3)考察嘧啶环不同取代基对杀菌活性影响; (4) 考察中间酮羰基还原为羟基亚甲基对杀菌活性影响(图 1). 本研究共计合成 36 个化合物, 化合物结构经 ${ }^{1} \mathrm{H}$ NMR、 ${ }^{13} \mathrm{C}$ NMR、HRMS 表征, 并进行了室内杀菌活性 测定.

\section{1 结果与讨论}

\subsection{2 -苯甲酰基嘧啶类化合物的合成}

2-苯甲酰基嘧啶类化合物的合成从易得的取代邻
氟苯乙腈出发，与取代的 2-甲砜基嘧啶在钠氢作用下发 生亲核取代反应，经过双氧水氧化处理后直接得到二芳 酮 $\mathbf{3}^{[5]}$. 3 与 $N$-甲基- $N-\mathrm{R}^{1}$ 取代甲酰基差弪胺在叔丁醇钾作 用下反应得到 4,4 经嗍氢化钠还原后得到目标化合物 5 (Scheme 1)，具体化合物见表 1 .<smiles>[X]c1cccc(F)c1CC#N</smiles>

$1 \mathrm{X}$<smiles>[R]Oc1nc(S(C)(O)O[Na])nc(O[R])c1[R]</smiles>

$2 \mathrm{~A} \sim 2 \mathrm{C}$<smiles>[R]Oc1nc(C(=O)c2c([X])cccc2F)nc(O[R])c1[R]</smiles><smiles>[R]Oc1nc(C(O)c2c([X])cccc2ON(C)C([R])=O)nc(O[R])c1[R]</smiles>

图式 1 2-苯甲酰基嘧啶类化合物合成路线

Scheme 1 Synthetic route of 2-benzoyl pyrimidine derivatives

表 1 2-苯甲酰基嘧啶类化合物

Table 1 Compound list of 2-benzoyl pyrimidine derivatives

\begin{tabular}{|c|c|c|c|}
\hline Compd. & $\mathrm{R}^{1}$ & Compd. & $\mathrm{R}^{1}$ \\
\hline 4АНа & $\mathrm{Ph}$ & $4 \mathrm{AFb}$ & $4-\mathrm{Cl}-\mathrm{C}_{6} \mathrm{H}_{4}$ \\
\hline 4AHb & 2-Me- $\mathrm{C}_{6} \mathrm{H}_{4}$ & $4 \mathrm{AFc}$ & 2-Cl- $\mathrm{C}_{6} \mathrm{H}_{4}$ \\
\hline 4АНс & $3-\mathrm{Me}-\mathrm{C}_{6} \mathrm{H}_{4}$ & 4AFd & $2-\mathrm{Me}-\mathrm{C}_{6} \mathrm{H}_{4}$ \\
\hline 4AHd & 4-Me- $\mathrm{C}_{6} \mathrm{H}_{4}$ & $4 \mathrm{AFe}$ & $3-\mathrm{Me}-\mathrm{C}_{6} \mathrm{H}_{4}$ \\
\hline 4АНе & $2-\mathrm{Cl}-\mathrm{C}_{6} \mathrm{H}_{4}$ & 4AFf & $2,3-\mathrm{Cl}_{2}-\mathrm{C}_{6} \mathrm{H}_{3}$ \\
\hline 4AHf & $3-\mathrm{Cl}-\mathrm{C}_{6} \mathrm{H}_{4}$ & 4AFg & $2,5-\mathrm{Cl}_{2}-\mathrm{C}_{6} \mathrm{H}_{3}$ \\
\hline 4AHg & $4-\mathrm{Cl}-\mathrm{C}_{6} \mathrm{H}_{4}$ & 4AFh & $2,6-\mathrm{Cl}_{2}-\mathrm{C}_{6} \mathrm{H}_{3}$ \\
\hline 4AHh & $4-\mathrm{MeO}-\mathrm{C}_{6} \mathrm{H}_{4}$ & $4 \mathrm{AFi}$ & 2-Thienyl \\
\hline $4 \mathbf{A H i}$ & $2-\mathrm{CF}_{3}-\mathrm{C}_{6} \mathrm{H}_{4}$ & $4 \mathrm{AFj}$ & 2-Furyl \\
\hline $\mathbf{4 A H j}$ & $3-\mathrm{CF}_{3}-\mathrm{C}_{6} \mathrm{H}_{4}$ & 4AFk & $\begin{array}{l}\text { 5-Chloro-1,3-trimethyl- } \\
1 H \text {-pyrazole-4 }\end{array}$ \\
\hline 4AHk & Cyclohexyl & 4AFI & $\begin{array}{l}\text { 2,2-Dichloro-1- } \\
\text { methylcyclopropanyl }\end{array}$ \\
\hline 4AHI & $2,3-\mathrm{Cl}_{2}-\mathrm{C}_{6} \mathrm{H}_{3}$ & 4AFm & $\begin{array}{l}\text { 2,2,3,3-Pentamethyl- } \\
\text { cyclopropanyl }\end{array}$ \\
\hline 4AHm & $2,6-\mathrm{Cl}_{2}-\mathrm{C}_{6} \mathrm{H}_{3}$ & 4ВНа & $2-\mathrm{Me}-\mathrm{C}_{6} \mathrm{H}_{4}$ \\
\hline 4AHn & $2,5-\mathrm{Cl}_{2}-\mathrm{C}_{6} \mathrm{H}_{3}$ & $4 \mathrm{CHa}$ & $2-\mathrm{Me}-\mathrm{C}_{6} \mathrm{H}_{4}$ \\
\hline 4АНо & 3-Me-thienyl-2 & $4 \mathrm{CFa}$ & $2,3-\mathrm{Cl}_{2}-\mathrm{C}_{6} \mathrm{H}_{3}$ \\
\hline 4АНр & $4-\mathrm{CF}_{3} \mathrm{O}-\mathrm{C}_{6} \mathrm{H}_{4}$ & $4 \mathrm{CFb}$ & $2,6-\mathrm{Cl}_{2}-\mathrm{C}_{6} \mathrm{H}_{3}$ \\
\hline 4AHq & 4-MeOOC- $\mathrm{C}_{6} \mathrm{H}_{4}$ & 5АНа & $2,6-\mathrm{Cl}_{2}-\mathrm{C}_{6} \mathrm{H}_{3}$ \\
\hline 4AFa & $3-\mathrm{Cl}-\mathrm{C}_{6} \mathrm{H}_{4}$ & $5 \mathrm{AHb}$ & $2,3-\mathrm{Cl}_{2}-\mathrm{C}_{6} \mathrm{H}_{3}$ \\
\hline
\end{tabular}


1.2 2-苯甲酰基嘧啶类化合物结构优化及室内杀菌活 性测定

\subsection{1 室内杀菌活性测定方法}

室内生测所用对照药剂苯菌酮按照文献[6]方法合 成, 嘧菌酯为商品药剂(先正达公司). 测试样品及对照 样品用 $N, N$-二甲基甲酰胺溶解配制成 $2 \%$ 乳油，用 $1 \%$ 吐 温-80 水溶液稀释成相应质量浓度后供试. 在整个生测 试验过程中, 菌种均为常年室内培养的敏感菌种; 测试 方法采用活体盆栽测试; 调查方法、要害分级及计算方 法参照《创制农药生物活性评价 SOP》，以病情指数计 算防治效果及 $\mathrm{EC}_{50} 、 \mathrm{EC}_{90}$ 值. 生测分为普篎、初篎和复 笁三个阶段. 普笁试验中设定笁选浓度为 $200 \mathrm{mg} / \mathrm{L}$, 䇻 选靶标为黄瓜白粉病、黄瓜霜霉病、黄瓜灰霉病、水稻 纹枯病. 在普篮试验中化合物对某个菌靶标的防效大于 $90 \%$ 的化合物进入对该菌靶标的初篎试验, 篮选浓度为 100、50、 $25 \mathrm{mg} / \mathrm{L}$. 在普笁试验中, 化合物在最低浓度 $25 \mathrm{mg} / \mathrm{L}$ 时对某个菌靶标的防效大于 $90 \%$ 进入复笁. 复 笁试验浓度根据化合物实际情况设置 5 7 个浓度梯度, 判断化合物对某种菌靶标的毒力效果.

\subsection{2 $\mathrm{R}^{1}$ 取代基优化及室内杀菌活性测定}

由于 $\mathrm{R}^{1}$ 取代基可选择范围比较大，首先固定嘧啶 环和中间苯环的取代基, 对 $\mathrm{R}^{1}$ 取代基进行结构优化. $\mathrm{R}^{1}$ 取代基以取代苯基为主，苯环取代基包括单取代或多取 代烷基、卤素、三氟甲基、碳酸酯基等. $\mathrm{R}^{1}$ 取代基的引 入通过 $\mathrm{R}^{1}$ 取代酰氯和 $N$-甲基盐酸差胺在碳酸钾/碳酸氢
钾作用下反应得到的 ${ }^{[7]}$. 我们从易得的 4,6-二甲氧基-2甲砜基嘧啶和 2-氟苯乙腈做为底物，合成一系列的 $\mathrm{R}^{1}$ 取代目标化合物，化合物见表 1 中 $\mathbf{4 A H a} \sim \mathbf{4 A H q}$.

普笁试验结果表明此类化合物对黄瓜霜霉病和黄 瓜白粉病具有很好防治效果, 但对黄瓜灰䨋病和水稻纹 枯病无效. 4AHb、4AHf 4AHh、4AHk、4AHm、4AHn 共 7 个化合物进入对黄瓜霜霉病的初篎试验; $4 \mathrm{AHa} 、$

4AHb、4AHe $\sim 4 \mathrm{AHg} 、 4 \mathrm{AHk} \sim 4 \mathrm{AHn} 、 4 \mathrm{AHp}$ 共 10 个 化合物进入对黄瓜白粉病的初篮试验(表 2).

表 $2 \mathrm{R}^{1}$ 取代化合物室内杀菌活性普篮实验

Table 2 Genaral fungicidal screening of compounds substituted with $\mathrm{R}^{1}$ group

\begin{tabular}{|c|c|c|c|c|c|}
\hline \multirow[b]{2}{*}{ 化合物 } & \multicolumn{2}{|c|}{ 防效/\% } & \multirow[b]{2}{*}{ 化合物 } & \multicolumn{2}{|c|}{ 防效/\% } \\
\hline & $\begin{array}{c}\text { 黄瓜霜 } \\
\text { 霉病 }\end{array}$ & $\begin{array}{c}\text { 黄瓜白 } \\
\text { 粉病 }\end{array}$ & & $\begin{array}{c}\text { 黄瓜霜 } \\
\text { 霉病 }\end{array}$ & $\begin{array}{c}\text { 黄瓜白 } \\
\text { 粉病 }\end{array}$ \\
\hline 4АНа & 67 & 100 & $\mathbf{4 A H j}$ & 11 & 11 \\
\hline 4AHb & 100 & 100 & 4AHk & 100 & 100 \\
\hline 4АHc & 0 & 83 & $4 \mathrm{AHI}$ & 22 & 100 \\
\hline 4AHd & 0 & 36 & 4AHm & 100 & 100 \\
\hline 4АHe & 67 & 100 & 4AHn & 100 & 100 \\
\hline 4AHf & 100 & 100 & 4АНо & 56 & 56 \\
\hline 4AHg & 100 & 100 & 4АНр & 22 & 100 \\
\hline 4AHh & 100 & 66 & $4 \mathrm{AHq}$ & 0 & 39 \\
\hline $4 \mathrm{AHr}$ & 0 & 0 & 嘧菌酯 & 100 & 100 \\
\hline
\end{tabular}

在初篎试验中 $4 \mathrm{AHI}$ 和 $4 \mathrm{AHn}$ 对黄瓜白粉病表现较 好, 进入复笁. $4 \mathrm{AHm}$ 由于其对黄瓜幼苗生长有一定致 畸影响被淘汰(表 3). 复篮试验结果表明, 4AHI 对黄瓜

表 3 化合物室内对黄瓜霜霉病和黄瓜白粉病初篮实验

Table 3 Interior primary screening of fungicidal activity against cucumber downy mildew and cucumber powdery mildew

\begin{tabular}{|c|c|c|c|c|c|c|c|}
\hline \multirow{2}{*}{ 化合物 } & \multirow{2}{*}{ 浓度/(mg・ $\left.\mathrm{L}^{-1}\right)$} & \multicolumn{2}{|c|}{ 防效/\% } & \multirow{2}{*}{ 化合物 } & \multirow{2}{*}{ 浓度/(mg・ $\left.\mathrm{L}^{-1}\right)$} & \multicolumn{2}{|c|}{ 防效/\% } \\
\hline & & 黄瓜霜霉病 & 黄瓜白粉病 & & & 黄瓜霜霉病 & 黄瓜白粉病 \\
\hline \multirow{3}{*}{ 4АНа } & 100 & - & 100 & \multirow{3}{*}{ 4AHh } & 100 & 100 & 78 \\
\hline & 50 & - & 87 & & 50 & 76 & 0 \\
\hline & 25 & - & 68 & & 25 & 55 & 0 \\
\hline \multirow{3}{*}{$4 \mathrm{AHb}$} & 100 & 100 & 100 & \multirow{3}{*}{ 4AHk } & 100 & 0 & 59 \\
\hline & 50 & 92 & 61 & & 50 & 0 & 37 \\
\hline & 25 & 13 & 39 & & 25 & 0 & 22 \\
\hline \multirow{3}{*}{ 4АHе } & 100 & - & 100 & \multirow{3}{*}{ 4AHI } & 100 & - & 100 \\
\hline & 50 & - & 82 & & 50 & - & 100 \\
\hline & 25 & - & 65 & & 25 & - & 100 \\
\hline \multirow{3}{*}{ 4AHf } & 100 & 100 & 59 & \multirow{3}{*}{ 4AHm } & 100 & 19 & 100 \\
\hline & 50 & 97 & 22 & & 50 & 7 & 100 \\
\hline & 25 & 72 & 0 & & 25 & 0 & 100 \\
\hline \multirow{3}{*}{ 4AHg } & 100 & 94 & 94 & \multirow{3}{*}{ 4AHn } & 100 & 15 & 100 \\
\hline & 50 & 79 & 11 & & 50 & 0 & 100 \\
\hline & 25 & 55 & 0 & & 25 & 0 & 98 \\
\hline \multirow{3}{*}{ 嘧菌酯 } & 100 & 100 & 100 & \multirow{3}{*}{ 苯菌酮 } & 100 & - & 100 \\
\hline & 50 & 100 & 100 & & 50 & - & 100 \\
\hline & 25 & 100 & 100 & & 25 & - & 100 \\
\hline
\end{tabular}


白粉病的 $\mathrm{EC}_{50}$ 和 $\mathrm{EC}_{90}$ 值分别为 0.95 和 $9.48 \mathrm{mg} / \mathrm{L}, \mathbf{4 A H n}$ 对黄瓜白粉病的 $\mathrm{EC}_{50}$ 和 $\mathrm{EC}_{90}$ 值分别为 0.08 和 14.14 $\mathrm{mg} / \mathrm{L}$, 与苯菌酮处在同一活性水平. 根据 $\mathrm{R}^{1}$ 取代目标 化合物结构及室内杀菌活性结果可以看出, $\mathrm{R}^{1}$ 取代基对 活性影响明显, 苯环上取代基优先顺序为: 烷基、卤 素 $\gg$ 强拉电子基团, 如三氟甲基、碳酸酯基等.

\subsection{3 苯环 $\mathrm{X}$ 取代基优化及室内杀菌活性测定}

在优化中间苯环 $\mathrm{X}$ 取代基过程中, 我们考虑到原料 及合成难易程度, 选择了 3 位和 6 位卤素取代化合物进 行结构优化. 3 位卤素取代我们尝试合成了氟、氯取代化 合物, 结果发现 3 位卤素取代的产物不稳定. 6 位卤素取 代化合物合成从易得的 2,6-二氟苯乙腈出发. $\mathrm{R}^{1}$ 取代基 选取活性表现较好的卤素或甲基取代的苯基作为取代 基. 另外根据电子等排原理, 增加了呋喃、噻吩、吡唑 及环丙基等取代基，具体化合物见表 1 中 $\mathbf{4 A F a} \sim$ 4AFm. 普篮试验结果表明 6-氟取代化合物对黄瓜白粉 病具有很好防治效果, 同样对黄瓜霜霉病、黄瓜灰霉病 和水稻纹枯病无效. $4 \mathrm{AFd} 、 4 \mathrm{AFi} \sim 4 \mathrm{AFk}$ 共 4 个化合物 进入对黄瓜白粉病的初篎试验.

初筛试验中 $4 \mathrm{AFd}$ 在 $25 \mathrm{mg} / \mathrm{L}$ 浓度下对黄瓜白粉病 的仍然保持 $100 \%$ 杀菌活性, 进入复篮(表 4). 复筛试验 结果表明 $4 \mathrm{AFd}$ 对黄瓜白粉病的 $\mathrm{EC}_{50}$ 和 $\mathrm{EC}_{90}$ 值分别为 2.26 和 $8.35 \mathrm{mg} / \mathrm{L}$, 较苯菌酮活性略低(表 5). 苯环取代 基优化结果表明苯环 6 位引入氟原子对杀菌活性影响不 显著, $\mathrm{R}^{1}$ 取代基引入杂环如呋喃、噻吩、吡唑及环丙基 等取代基对活性没有明显提高.

表 4 化合物室内杀菌活性初篮实验结果

Table 4 Primary screening result of interior fungicidal activity

\begin{tabular}{|c|c|c|c|c|c|}
\hline 化合物 & $\begin{array}{c}\text { 浓度/ } \\
\left(\mathrm{mg} \cdot \mathrm{L}^{-1}\right)\end{array}$ & $\frac{\text { 防效 } / \%}{\text { 黄瓜白粉病 }}$ & 化合物 & $\begin{array}{c}\text { 浓度/ } \\
\left(\mathrm{mg} \cdot \mathrm{L}^{-1}\right)\end{array}$ & $\begin{array}{c}\text { 防效 } / \% \\
\text { 黄瓜白粉病 }\end{array}$ \\
\hline \multirow{3}{*}{ 4AFd } & 100 & 100 & \multirow{3}{*}{$\mathbf{4 A F j}$} & 100 & 100 \\
\hline & 50 & 100 & & 50 & 78 \\
\hline & 25 & 100 & & 25 & 44 \\
\hline \multirow{3}{*}{$\mathbf{4 A F i}$} & 100 & 100 & \multirow{3}{*}{ 4AFk } & 100 & 0 \\
\hline & 50 & 100 & & 50 & 0 \\
\hline & 25 & 79 & & 25 & 0 \\
\hline \multirow{3}{*}{ 嘧菌酯 } & 100 & 100 & \multirow{3}{*}{ 苯菌酮 } & 100 & 100 \\
\hline & 50 & 100 & & 50 & 100 \\
\hline & 25 & 100 & & 25 & 100 \\
\hline
\end{tabular}

1.2.4 嘧啶环取代基 $\mathrm{R}^{2} 、 \mathrm{R}^{3}$ 优化及室内杀菌活性测 定

嘧啶环取代基优化, 首先考察了 $\mathrm{R}^{2}$ 添加取代基对 活性影响，合成了 5 位甲基取代化合物，保持 4,6 位双甲 氧基, 化合物见表 1 中 $4 \mathrm{BHa}$. 同时通过延长烷氧基链 考察 $\mathrm{R}^{3}$ 取代基对活性影响, 边链 $\mathrm{R}^{1}$ 选取了篮选出的高 活性化合物边链, 中间环以苯环和 6-F 取代苯环为主, 化合物见表 1 中 $4 \mathrm{CHa} 、 4 \mathrm{CFa}$ 及 $4 \mathrm{CFb}$. 普篮试验结果
表 5 化合物对黄瓜白粉病复篮

Table 5 Secondary screening of interior fungicidal activity against cucumber powdery mildew

\begin{tabular}{|c|c|c|c|c|c|}
\hline 化合物 & $\begin{array}{c}\mathrm{EC}_{50} / \\
\left(\mathrm{mg} \cdot \mathrm{L}^{-1}\right)\end{array}$ & $\begin{array}{c}\mathrm{EC}_{90} / \\
\left(\mathrm{mg} \cdot \mathrm{L}^{-1}\right)\end{array}$ & $\begin{array}{c}\text { 毒力回归 } \\
\text { 曲线 }\end{array}$ & \multicolumn{2}{|c|}{ 相关系数 $r$ 标准误差 } \\
\hline 4AHI & 0.95 & 9.48 & $\begin{array}{c}y=5.0298+ \\
1.2812 x\end{array}$ & 0.9726 & 0.1769 \\
\hline 4AHn & 0.08 & 14.14 & $\begin{array}{c}y=5.6252+ \\
0.5705 x\end{array}$ & 0.9967 & 0.0455 \\
\hline 4AFd & 2.26 & 8.35 & $\begin{array}{c}y=4.2024+ \\
2.2553 x\end{array}$ & 0.9994 & 0.0536 \\
\hline 苯菌酮 & 0.14 & 2.13 & $\begin{array}{c}y=5.9262+ \\
1.0790 x\end{array}$ & 0.9715 & 0.1519 \\
\hline
\end{tabular}

表明, 改变嘧啶环上的取代基无论是 5 位增加甲基还是 增长烷氧基边链都会使化合物杀菌活性大大降低.

\subsection{5 羰基结构优化及室内杀菌活性测定}

酮羰基结构优化, 我们将酮羰基还原为羟基亚甲 基. 选取 4AHm 和 4AHI 作为底物, 通过硼氢化钠还原 分别得到化合物 $5 \mathrm{AHa}$ 和 $5 \mathrm{AHb}$. 室内杀菌活性初篮结 果表明, 酮羰基一旦被还原为羟基亚甲基后，杀菌活性 完全丧失, 可能的原因是氢键受体变为氢键给体所致.

\section{2 结论}

新型嘧啶甲酰基苯基羟胺类化合物是从具有自主 知识产权的 2-嘧啶氧基- $N$-芳基苄胺类化合物衍生得到 的先导化合物. 此类先导化合物具有全新的骨架结构, 并对黄瓜白粉病和黄瓜霜霉病表现出一定的杀菌活性. 本文主要讨论了此类化合物的合成, 并对其进行了杀菌 活性篎选. 针对先导化合物不同的部位进行了以下几个 方面结构优化工作: (1)改变 $\mathrm{R}^{1}$ 取代基; (2)改变中间苯环 取代基; (3)考察嘧啶环不同取代基对杀菌活性影响; (4) 考察中间酮羰基还原为羟基亚甲基对杀菌活性影响, 共 计合成 36 个化合物. 根据生测结果推断, $\mathrm{R}^{1}$ 取代基以 2 位卤素或烷基取代苯环或杂环活性最好; 中间苯环 6 位 引入氟原子, 活性不受影响, 其它位置取代活性有待进 一步验证; 嘧啶环 4,6 位甲氧基取代活性较好, 5 位甲基 取代活性大大降低; 羊基被还原为羟基后活性消失.

\section{3 实验部分}

\section{1 仪器与试剂}

Agilent 400-MR DD2 核磁共振波谱仪、Agilent 6120 Quadrupole LC-MS 系统、Shimadzu LCMS-2010EV 质谱 仪、Shimadzu LC-20AD 高效液相色谱仪、Diamonsil C18 柱. WPSHZ-500 全自动喷雾塔(南京农业机械研究所). HPLC 采用乙腈、甲醇、冰醋酸均为色谱纯, 购自 Tedia 公司; 水为新蒸的二次重蒸水. 其他试剂均为市售分析 纯试剂. 


\section{2 实验方法}

\subsection{1 化合物 4 通用合成方法}

以化合物 $\mathbf{4 A H}$ 为例. 在氮气保护下, 在 $100 \mathrm{~mL}$ 三 口烧瓶中加入 2 -氟苯乙腈 $(\mathbf{1 H})(1.48 \mathrm{~g}, 11 \mathrm{mmol})$ 与 30 $\mathrm{mL}$ 无水 $\mathrm{DMF}, 0{ }^{\circ} \mathrm{C}$ 下, 缓缓加入 $\mathrm{NaH}(880 \mathrm{mg}, 22$ $\mathrm{mmol}, 60 \%$ ), 加完后搅拌 $30 \mathrm{~min}$, 加入 4,6-二甲氧基-2甲砜基嘧啶(2a) (2.18 g, $10 \mathrm{mmol})$, 升至室温反应 $24 \mathrm{~h}$. 在冰浴下, 向反应液中缓慢滴加 $15 \mathrm{~mL}$ 双氧水, 反应放 热剧烈. 滴加完毕后, 继续室温反应过夜. 反应液倒入 $100 \mathrm{~mL}$ 冰水中, 静置、过滤, 滤饼经水洗、干燥后, 用 乙酸乙酯/石油醚重结晶得到 (4,6-二甲氧基-2-嘧啶 基)(2-氟苯基)甲酮(3AH) $2.14 \mathrm{~g}$, 收率 $82 \%$.

将羟胺 $(1 \mathrm{mmol})$ 溶于 $20 \mathrm{~mL} \mathrm{DMF}$, 降温至 $0{ }^{\circ} \mathrm{C}$, 加 入叔丁醇钾 $(1.1 \mathrm{mmol})$, 搅拌 $30 \mathrm{~min}$ 后, 加入化合物 3AH $(1.1 \mathrm{mmol})$, 升温至 $40{ }^{\circ} \mathrm{C}$ 反应 $8 \mathrm{~h}$. 反应液倒入 $100 \mathrm{~mL}$ 饱和食盐水, 乙酸乙酯 $(30 \mathrm{~mL} \times 3)$ 萃取, 有机相 经水洗、干燥、旋干后, 粗品经柱层析 $[V$ (乙酸乙酯) : $V($ 石油醚 $)=1: 5]$ 分离后得化合物 $\mathbf{4 A H}$, 收率 $40 \%$ $80 \%$.

$N$-[2-(4,6-二甲氧基嘧啶基-2-甲酰基)苯氧基]- $N$-甲 基苯甲酰胺(4AHa): m.p. $95 \sim 97{ }^{\circ} \mathrm{C}$; HPLC: $98.3 \%$; ${ }^{1} \mathrm{H}$ NMR (DMSO- $\left.d_{6}, 300 \mathrm{MHz}\right) \delta: 7.76 \sim 7.64(\mathrm{~m}, 2 \mathrm{H}, \mathrm{Ph}-\mathrm{H})$, $7.51 \sim 7.30$ (m, 6H, Ph-H), 7.30 7.22 (m, 1H, Ph-H), 6.39 (s, $1 \mathrm{H}, \mathrm{Py}-\mathrm{H}), 3.79$ (s, $\left.6 \mathrm{H}, \mathrm{OCH}_{3}\right), 3.10\left(\mathrm{~s}, 3 \mathrm{H}, \mathrm{NCH}_{3}\right) ;{ }^{13} \mathrm{C}$ NMR $\left(\mathrm{CDCl}_{3}, 101 \mathrm{MHz}\right) \delta$ : 190.69, 171.96, 171.46, $162.05,156.37,134.00,132.64,131.37,131.21,128.00$, $127.96,125.05,123.19,112.47,91.13,54.31,35.50$. HRMS calcd for $\mathrm{C}_{21} \mathrm{H}_{19} \mathrm{~N}_{3} \mathrm{NaO}_{5}[\mathrm{M}+\mathrm{Na}]^{+}$416.1217, found 416.1224 .

$N$-[2-(4,6-二甲氧基嘧啶基-2-甲酰基)苯氧基]- $N$,2二甲基苯甲酰胺 $(\mathbf{4 A H b})$ : m.p. $107 \sim 108{ }^{\circ} \mathrm{C}$; HPLC: 99.0\%; ${ }^{1} \mathrm{H}$ NMR $\left(\mathrm{CDCl}_{3}, 300 \mathrm{MHz}\right) \delta: 7.67$ (dd, $J=7.8$, $1.7 \mathrm{~Hz}, 1 \mathrm{H}, \mathrm{Ph}-\mathrm{H}), 7.57 \sim 7.45$ (m, 1H, Ph-H), $7.21 \sim 7.09$ (m, 3H, Ph-H), 7.09 6.96 (m, 3H, Ph-H), $6.11(\mathrm{~s}, 1 \mathrm{H}$, Py-H), 3.83 (s, 6H, $\left.\mathrm{OCH}_{3}\right), 3.24$ (s, 3H, $\left.\mathrm{NCH}_{3}\right), 2.16$ (s, $\left.3 \mathrm{H}, \mathrm{Ph}-\mathrm{CH}_{3}\right) ;{ }^{13} \mathrm{C} \mathrm{NMR}\left(\mathrm{CDCl}_{3}, 101 \mathrm{MHz}\right) \delta: 190.84$, $172.35,171.42,161.75,156.26,134.86,133.82,133.57$, $131.14,130.16,129.51,125.91,125.78,125.15,123.13$, 112.31, 91.37, 54.30, 34.73, 19.07. HRMS calcd for $\mathrm{C}_{22} \mathrm{H}_{21} \mathrm{~N}_{3} \mathrm{O}_{5}[\mathrm{M}+\mathrm{Na}]^{+}$430.1373, found 430.1377 .

$N$-[2-(4,6-二甲氧基嘧啶基-2-甲酰基)苯氧基]- $N, 3$ 二甲基苯甲酰胺(4AHc): m.p. $115 \sim 116{ }^{\circ} \mathrm{C}$; HPLC: 98.7\%; ${ }^{1} \mathrm{H}$ NMR $\left(\mathrm{CDCl}_{3}, 400 \mathrm{MHz}\right) \delta: 7.76$ (dd, $J=7.6$, $1.5 \mathrm{~Hz}, 1 \mathrm{H}, \mathrm{Ph}-\mathrm{H}), 7.57 \sim 7.51$ (m, 1H, Ph-H), 7.34 (s, 1H, Ph-H), 7.25 (d, $J=7.5$ Hz, 1H, Ph-H), $7.21 \sim 7.09$ (m, 4H,
Ph-H), 6.08 (s, 1H, Py-H), 3.84 (s, 6H, $\mathrm{OCH}_{3}$ ), 3.23 (s, 3H, $\left.\mathrm{NCH}_{3}\right), 2.28$ (s, 3H, Ph- $\left.\mathrm{CH}_{3}\right) ;{ }^{13} \mathrm{C}$ NMR $\left(\mathrm{CDCl}_{3}, 101\right.$ MHz) $\delta: 190.69,172.20,171.47,162.06,156.53,137.80$, 133.97, 132.65, 131.96, 131.33, 128.69, 127.78, 125.68, 124.90, 123.08, 112.43, 91.14, 54.29, 35.66, 21.16. HRMS calcd for $\mathrm{C}_{22} \mathrm{H}_{22} \mathrm{~N}_{3} \mathrm{O}_{5}[\mathrm{M}+\mathrm{H}]^{+}$408.1554, found 408.1569 .

$N$-[2-(4,6-二甲氧基嘧啶基-2-甲酰基)苯氧基]- $N$,4二甲基苯甲酰胺(4AHd): m.p. $186 \sim 187{ }^{\circ} \mathrm{C}$; HPLC: $89.7 \%$; ${ }^{1} \mathrm{H}$ NMR (DMSO- $\left.d_{6}, 400 \mathrm{MHz}\right) \delta: 7.74$ (dd, $J=$ 7.6, $1.5 \mathrm{~Hz}, 1 \mathrm{H}, \mathrm{Ph}-\mathrm{H}), 7.72 \sim 7.67$ (m, 1H, Ph-H), 7.38 7.30 (m, 3H, Ph-H), $7.29 \sim 7.23$ (m, 1H, Ph-H), 7.15 (d, $J=8.0 \mathrm{~Hz}, 2 \mathrm{H}, \mathrm{Ph}-\mathrm{H}), 6.37$ (s, $1 \mathrm{H}, \mathrm{Py}-\mathrm{H}), 3.79$ (s, $6 \mathrm{H}$, $\mathrm{OCH}_{3}$ ), 3.08 (s, 3H, $\left.\mathrm{NCH}_{3}\right), 2.29$ (s, 3H, Ph- $\left.\mathrm{CH}_{3}\right) ;{ }^{13} \mathrm{C}$ $\mathrm{NMR}\left(\mathrm{CDCl}_{3}, 101 \mathrm{MHz}\right) \delta: 190.80,172.07,171.45$, $162.11,156.44,141.68,134.01,131.30,129.67,128.58$, 128.19, 125.71, 123.11, 112.46, 91.10, 54.27, 35.51, 21.39. HRMS calcd for $\mathrm{C}_{22} \mathrm{H}_{21} \mathrm{~N}_{3} \mathrm{NaO}_{5}[\mathrm{M}+\mathrm{Na}]^{+}$430.1373, found 430.1355 .

2-氯- $N$-[2-(4,6-二甲氧基嘧啶基-2-甲酰基)苯氧 基]- $\mathrm{N}$-甲基苯甲酰胺(4AHe): m.p. 98 99 ${ }^{\circ} \mathrm{C}$; HPLC: $98.0 \% ;{ }^{1} \mathrm{H} \mathrm{NMR}\left(\mathrm{CDCl}_{3}, 300 \mathrm{MHz}\right) \delta: 7.72 \sim 7.62(\mathrm{~m}, 1 \mathrm{H}$, Ph-H), $7.58 \sim 7.46(\mathrm{~m}, 1 \mathrm{H}, \mathrm{Ph}-\mathrm{H}), 7.26 \sim 7.07$ (m, 6H, Ph-H), 6.13 (s, 1H, Py-H), 3.85 (s, 6H, OCH $)_{3}, 3.25$ (s, 3H, $\left.\mathrm{NCH}_{3}\right) ;{ }^{13} \mathrm{C}$ NMR $\left(\mathrm{CDCl}_{3}, 101 \mathrm{MHz}\right) \delta: 190.90,171.47$, $169.53,161.66,156.02,133.88,133.64,131.11,130.66$, $130.60,129.46,127.22,126.38,126.09,123.40,112.51$, 91.44, 54.30, 34.68. HRMS calcd for $\mathrm{C}_{21} \mathrm{H}_{18} \mathrm{ClN}_{3} \mathrm{NaO}_{5}$ $[\mathrm{M}+\mathrm{Na}]^{+} 450.0827$, found 450.0811 .

3-氯- $N$-[2-(4,6-二甲氧基嘧啶基-2-甲酰基)苯氧 基]- $\mathrm{N}$-甲基苯甲酰胺(4AHf): m.p. $93 \sim 95{ }^{\circ} \mathrm{C}$; HPLC: $97.2 \%$; ${ }^{1} \mathrm{H}$ NMR (DMSO- $\left.d_{6}, 300 \mathrm{MHz}\right) \delta: 7.75 \sim 7.65(\mathrm{~m}$, 2H, Ph-H), $7.55 \sim 7.49$ (m, 1H, Ph-H), 7.47 (d, $J=1.0 \mathrm{~Hz}$, 1H, Ph-H), 7.44 7.35 (m, 3H, Ph-H), 7.30 7.23 (m, 1H, Ph-H), 6.39 (s, 1H, Py-H), 3.80 (s, 6H, OCH $), 3.12$ (s, 3H, $\left.\mathrm{NCH}_{3}\right) ;{ }^{13} \mathrm{C} \mathrm{NMR}\left(\mathrm{CDCl}_{3}, 101 \mathrm{MHz}\right) \delta: 190.48,171.40$, $170.45,161.87,156.10,134.38,134.13,133.98,131.54$, $131.25,129.27,128.29,125.91,125.77,123.35,112.27$, 91.28, 54.52, 35.44. HRMS calcd for $\mathrm{C}_{21} \mathrm{H}_{18} \mathrm{ClN}_{3} \mathrm{NaO}_{5}$ $[\mathrm{M}+\mathrm{Na}]^{+} 450.0827$, found 450.0820 .

4-氯- $N$-[2-(4,6-二甲氧基嘧啶基-2-甲酰基)苯氧 基]- $\mathrm{N}$-甲基苯甲酰胺(4AHg): m.p. $123 \sim 124{ }^{\circ} \mathrm{C}$; HPLC: 98.7\%; ${ }^{1} \mathrm{H}$ NMR (DMSO- $\left.d_{6}, 300 \mathrm{MHz}\right) \delta: 7.76 \sim 7.66(\mathrm{~m}$, $2 \mathrm{H}, \mathrm{Ph}-\mathrm{H}), 7.44 \sim 7.37$ (m, 5H, Ph-H), $7.31 \sim 7.23(\mathrm{~m}, 1 \mathrm{H}$, Ph-H), 6.37 (s, 1H, Py-H), 3.79 (s, 6H, OCH 3 ), 3.11 (s, 3H, 
$\left.\mathrm{NCH}_{3}\right) ;{ }^{13} \mathrm{C} \mathrm{NMR}\left(\mathrm{CDCl}_{3}, 101 \mathrm{MHz}\right) \delta: 190.52,171.41$, 170.82, 161.94, 156.11, 137.48, 134.02, 131.58, 130.91, $129.60,128.28,125.73,123.33,112.24,91.12,54.44$, 35.33. HRMS calcd for $\mathrm{C}_{21} \mathrm{H}_{18} \mathrm{ClN}_{3} \mathrm{NaO}_{5}[\mathrm{M}+\mathrm{Na}]^{+}$ 450.0827 , found 450.0828

$N$-[2-(4,6-二甲氧基嘧啶基-2-甲酰基)苯氧基]-4-甲 氧基- $N$ - 甲基苯甲酰胺 (4AHh): m.p. $103 \sim 105{ }^{\circ} \mathrm{C}$; HPLC: $98.4 \%$; ${ }^{1} \mathrm{H}$ NMR (DMSO- $\left.d_{6}, 300 \mathrm{MHz}\right) \delta: 7.81 \sim$ $7.64(\mathrm{~m}, 2 \mathrm{H}, \mathrm{Ph}-\mathrm{H}), 7.43$ (d, $J=8.8 \mathrm{~Hz}, 2 \mathrm{H}, \mathrm{Ph}-\mathrm{H}), 7.37$ (d, $J=8.3 \mathrm{~Hz}, 1 \mathrm{H}, \mathrm{Ph}-\mathrm{H}), 7.33 \sim 7.21$ (m, 1H, Ph-H), 6.89 (d, $J=8.9 \mathrm{~Hz}, 2 \mathrm{H}, \mathrm{Ph}-\mathrm{H}$ ), 6.37 (s, 1H, Py-H), 3.78 (s, 6H, Py- $\mathrm{OCH}_{3}$ ), 3.76 (s, $3 \mathrm{H}, \mathrm{PhOCH}_{3}$ ), 3.06 (s, $\left.3 \mathrm{H}, \mathrm{NCH}_{3}\right) ;{ }^{13} \mathrm{C}$ NMR $\left(\mathrm{CDCl}_{3}, 101 \mathrm{MHz}\right) \delta$ : 190.78, 171.61, 171.47, $162.15,161.99,156.49,134.09,131.36,130.44,125.67$, $124.56,123.10,113.26,112.50,91.04,55.20,54.29,35.62$. HRMS calcd for $\mathrm{C}_{22} \mathrm{H}_{21} \mathrm{~N}_{3} \mathrm{NaO}_{6}[\mathrm{M}+\mathrm{Na}]^{+}$446.1323, found 446.1332

$\mathrm{N}$-[2-(4,6-二甲氧基嘧啶基-2-甲酰基)苯氧基]- $\mathrm{N}$-甲 基-2-三氟甲基苯甲酰胺 (4AHi): m.p. $107 \sim 109{ }^{\circ} \mathrm{C}$; HPLC: $99.4 \%$; ${ }^{1} \mathrm{H}$ NMR (DMSO- $\left.d_{6}, 300 \mathrm{MHz}\right) \delta: 7.79 \sim$ $7.33(\mathrm{~m}, 7 \mathrm{H}, \mathrm{Ph}-\mathrm{H}), 7.29 \sim 7.20(\mathrm{~m}, 1 \mathrm{H}, \mathrm{Ph}-\mathrm{H}), 6.38(\mathrm{~s}$, $1 \mathrm{H}, \mathrm{Py}-\mathrm{H}), 3.77$ (s, $\left.6 \mathrm{H}, \mathrm{OCH}_{3}\right), 3.09$ (s, $\left.3 \mathrm{H}, \mathrm{NCH}_{3}\right) ;{ }^{13} \mathrm{C}$ NMR $\left(\mathrm{CDCl}_{3}, 101 \mathrm{MHz}\right) \delta: 190.94,171.42,171.00$, $161.43,155.68,133.53,132.63,131.23,129.64,126.99$ (q, $J=64.4,32.2 \mathrm{~Hz}$ ), 126.35 (q, $J=4.5 \mathrm{~Hz}), 126.12,126.17$, 126.16, 123.39, 123.29 (q, $J=273.8 \mathrm{~Hz}), 112.15,91.38$, 54.22, 34.68. HRMS calcd for $\mathrm{C}_{22} \mathrm{H}_{18} \mathrm{~F}_{3} \mathrm{~N}_{3} \mathrm{NaO}_{5}[\mathrm{M}+\mathrm{Na}]^{+}$ 484.1091, found 484.1096.

$N$-[2-(4,6-二甲氧基嘧啶基-2-甲酰基)苯氧基]- $N$-甲 基-3-三氟甲基苯甲酰胺 $(\mathbf{4 A H j}):$ m.p. $96 \sim 98{ }^{\circ} \mathrm{C}$; HPLC: 95.9\%; ${ }^{1} \mathrm{H}$ NMR (DMSO- $\left.d_{6}, 300 \mathrm{MHz}\right) \delta: 7.83$ (d, $J=7.8$ $\mathrm{Hz}, 1 \mathrm{H}, \mathrm{Ph}-\mathrm{H}), 7.76$ (s, 1H, Ph-H), 7.74 7.66 (m, 3H, $\mathrm{Ph}-\mathrm{H}), 7.63 \sim 7.55(\mathrm{~m}, 1 \mathrm{H}, \mathrm{Ph}-\mathrm{H}) 7.49 \sim 7.43(\mathrm{~m}, 1 \mathrm{H}$, Ph-H), $7.31 \sim 7.24$ (m, 1H, Ph-H), 6.38 (s, 1H, Py-H), 3.78 (s, 6H, $\left.\mathrm{OCH}_{3}\right), 3.15\left(\mathrm{~s}, 3 \mathrm{H}, \mathrm{NCH}_{3}\right) ;{ }^{13} \mathrm{C} \mathrm{NMR}\left(\mathrm{CDCl}_{3}, 101\right.$ MHz) $\delta: 190.31,171.49,170.45,161.79,156.03,133.97$, 133.46, 131.70, 131.01 (q, $J=1.1 \mathrm{~Hz}), 130.66$ (q, $J=32.9$ $\mathrm{Hz}), 128.52,127.81$ (q, $J=3.6 \mathrm{~Hz}), 125.71,125.31$ (q, $J=$ $3.9 \mathrm{~Hz}), 123.48$ (q, $J=272.5 \mathrm{~Hz}), 123.39,112.12,91.22$, 54.29, 35.34. HRMS calcd for $\mathrm{C}_{22} \mathrm{H}_{18} \mathrm{~F}_{3} \mathrm{~N}_{3} \mathrm{NaO}_{5}[\mathrm{M}+\mathrm{Na}]^{+}$ 484.1091 , found 484.1107 .

$N$-[2-(4,6-二甲氧基嘧啶基-2-甲酰基)苯氧基]- $N$-甲 基环已基甲酰胺(4AHk): m.p. $96 \sim 97{ }^{\circ} \mathrm{C}$; HPLC: 98.5\%; ${ }^{1} \mathrm{H}$ NMR (DMSO- $\left.d_{6}, 300 \mathrm{MHz}\right) \delta: 7.79(\mathrm{dd}, J=7.6,1.7$ $\mathrm{Hz}, 1 \mathrm{H}, \mathrm{Ph}-\mathrm{H}$ ), $7.74 \sim 7.63$ (m, 1H, Ph-H), $7.34 \sim 7.25$ (m,
1H, Ph-H), 7.21 (d, J=8.2 Hz, 1H, Ph-H), $6.46(\mathrm{~s}, 1 \mathrm{H}$, Py-H), 3.86 (s, 6H, $\left.\mathrm{OCH}_{3}\right), 2.96$ (s, 3H, $\left.\mathrm{NCH}_{3}\right), 2.33 \sim$ $2.19\left(\mathrm{~m}, 1 \mathrm{H}\right.$, cyclo- $\left.\mathrm{C}_{6} \mathrm{H}_{11}-1-\mathrm{H}\right), 1.67 \sim 1.43(\mathrm{~m}, 5 \mathrm{H}$, cyclo- $\left.\mathrm{C}_{6} \mathrm{H}_{11}\right), 1.29 \sim 0.96\left(\mathrm{~m}, 5 \mathrm{H}\right.$, cyclo- $\left.\mathrm{C}_{6} \mathrm{H}_{11}\right) ;{ }^{13} \mathrm{C}$ NMR $\left(\mathrm{CDCl}_{3}, 101 \mathrm{MHz}\right) \delta: 190.78,179.18,171.58,162.30$, $156.91,134.17,131.45,125.51,123.17,112.06,91.14$, $54.35,40.20,34.18,28.54,25.56,25.30$. HRMS calcd for $\mathrm{C}_{21} \mathrm{H}_{25} \mathrm{~N}_{3} \mathrm{O}_{5}[\mathrm{M}+\mathrm{Na}]^{+} 422.1686$, found 422.1716 .

2,3-二氯- $N$-[2-(4,6-二甲氧基嘧啶基-2-甲酰基)苯氧 基]- $N$-甲基苯甲酰胺(4AHI): oil; HPLC: 99.5\%; ${ }^{1} \mathrm{H}$ NMR (DMSO- $\left.d_{6}, 300 \mathrm{MHz}\right) \delta: 7.75 \sim 7.56$ (m, 3H, Ph-H), 7.44 (d, $J=8.2 \mathrm{~Hz}, 1 \mathrm{H}, \mathrm{Ph}-\mathrm{H}), 7.38 \sim 7.16$ (m, 3H, Ph-H), 6.40 (s, 1H, Py-H), $3.81\left(\mathrm{~s}, 6 \mathrm{H}, \mathrm{OCH}_{3}\right), 3.49 \sim 3.21(\mathrm{~m}, 3 \mathrm{H}$, $\left.\mathrm{NCH}_{3}\right) ;{ }^{13} \mathrm{C}$ NMR $\left(\mathrm{CDCl}_{3}, 101 \mathrm{MHz}\right) \delta: 190.77,171.44$, $168.82,161.58,155.79,135.96,133.70,133.36,131.31$, $131.24,129.09,127.29,126.17,125.09,123.62,112.46$, 91.52, 54.37, 34.33. HRMS calcd for $\mathrm{C}_{21} \mathrm{H}_{17} \mathrm{Cl}_{2} \mathrm{~N}_{3} \mathrm{NaO}_{5}$ $[\mathrm{M}+\mathrm{Na}]^{+}$484.0437, found 484.0449.

2,6-二氯- $N$-[2-(4,6-二甲氧基嘧啶基-2-甲酰基)苯氧 基]- $N$-甲基苯甲酰胺(4AHm): m.p. $123 \sim 125{ }^{\circ} \mathrm{C}$; HPLC: 98.1\%; ${ }^{1} \mathrm{H}$ NMR (DMSO, $\left.300 \mathrm{MHz}\right) \delta: 7.71 \sim 7.53(\mathrm{~m}$, 2H, Ph-H), $7.41 \sim 7.29$ (m, 4H, Ph-H), 7.24 (td, $J=7.5 \mathrm{~Hz}$, $1 \mathrm{H}, \mathrm{Ph}-\mathrm{H}), 6.43$ (s, 1H, Py-H), 3.80 (s, 6H, $\left.\mathrm{OCH}_{3}\right), 3.23$ (s, $\left.3 \mathrm{H}, \mathrm{NCH}_{3}\right) ;{ }^{13} \mathrm{C}$ NMR $\left(\mathrm{CDCl}_{3}, 101 \mathrm{MHz}\right) \delta: 191.20$, $171.46,167.43,161.28,155.57,133.57,133.46,131.46$, $130.91,130.64,127.57,126.53,123.68,113.22,91.44$, 54.35, 34.46. HRMS calcd for $\mathrm{C}_{21} \mathrm{H}_{17} \mathrm{Cl}_{2} \mathrm{~N}_{3} \mathrm{NaO}_{5}[\mathrm{M}+$ $\mathrm{Na}]^{+}$484.0437, found 484.0444 .

2,5-二氯- $N$-[2-(4,6-二甲氧基嘧啶基-2-甲酰基)苯氧 基]- $\mathrm{N}$-甲基苯甲酰胺(4AHn): m.p. $126 \sim 128{ }^{\circ} \mathrm{C}$; HPLC: 99.7\%; ${ }^{1} \mathrm{H}$ NMR $\left(\mathrm{CDCl}_{3}, 400 \mathrm{MHz}\right) \delta: 7.67(\mathrm{~d}, J=7.6 \mathrm{~Hz}$, 1H, Ph-H), $7.59 \sim 7.49$ (m, 1H, Ph-H), $7.24 \sim 7.05$ (m, 5H, Ph-H), 6.13 (s, 1H, Py-H), 3.86 (s, 6H, $\mathrm{OCH}_{3}$ ), 3.27 (s, 3H, $\left.\mathrm{NCH}_{3}\right) ;{ }^{13} \mathrm{C}$ NMR $\left(\mathrm{CDCl}_{3}, 101 \mathrm{MHz}\right) \delta: 190.93,171.44$, $168.10,161.39,155.66,135.28,133.58,132.44,131.19$, 130.71, 130.64, 128.97, 127.13, 126.30, 123.63, 112.34, 91.62, 54.36, 34.49. HRMS calcd for $\mathrm{C}_{21} \mathrm{H}_{17} \mathrm{Cl}_{2} \mathrm{~N}_{3} \mathrm{NaO}_{5}$ $[\mathrm{M}+\mathrm{Na}]^{+}$484.0437, found 484.0434.

$N$-[2-(4,6-二甲氧基嘧啶基-2-甲酰基)苯氧基]- $N, 3$ 二甲基噻吩-2-甲酰胺(4AHo): m.p. $119 \sim 121{ }^{\circ} \mathrm{C}$; HPLC: 98.6\%; ${ }^{1} \mathrm{H}$ NMR $\left(\mathrm{CDCl}_{3}, 400 \mathrm{MHz}\right) \delta: 7.81$ (dd, $J=7.6$, $1.7 \mathrm{~Hz}, 1 \mathrm{H}, \mathrm{Ph}-\mathrm{H}), 7.54 \sim 7.47$ (m, 1H, Ph-H), $7.25 \sim 7.13$ $(\mathrm{m}, 3 \mathrm{H}, \mathrm{Ar}-\mathrm{H}), 6.80(\mathrm{~d}, J=5.0 \mathrm{~Hz}, 1 \mathrm{H}$, Thiophene-H), 6.08 (s, 1H, Py-H), 3.86 (d, $\left.J=2.0 \mathrm{~Hz}, 6 \mathrm{H}, \mathrm{OCH}_{3}\right), 3.16$ (d, $\left.J=2.0 \mathrm{~Hz}, 3 \mathrm{H}, \quad \mathrm{NCH}_{3}\right), 2.48\left(\mathrm{~s}, 3 \mathrm{H}\right.$, Thiophene- $\left.\mathrm{CH}_{3}\right)$; 
${ }^{13} \mathrm{C}$ NMR $\left(\mathrm{CDCl}_{3}, 101 \mathrm{MHz}\right) \delta: 190.91,171.53,166.02$, $162.12,156.20,147.32,133.98,131.24,130.66,129.76$, 126.10, 123.94, 123.49, 112.80, 91.18, 54.35, 34.93, 16.87 . HRMS calcd for $\mathrm{C}_{20} \mathrm{H}_{19} \mathrm{~N}_{3} \mathrm{O}_{5} \mathrm{NaS}[\mathrm{M}+\mathrm{Na}]^{+}$436.0938, found 436.0940 .

$N$-[2-(4,6-二甲氧基嘧啶基-2-甲酰基)苯氧基]- $N$-甲 基-4-三氟甲氧基苯甲酰胺(4AHp): oil; HPLC: $93.6 \% ;{ }^{1} \mathrm{H}$ NMR (DMSO- $\left.d_{6}, 300 \mathrm{MHz}\right) \delta$ : $7.76 \sim 7.66(\mathrm{~m}, 2 \mathrm{H}, \mathrm{Ph}-\mathrm{H})$, $7.60 \sim 7.54$ (m, 2H, Ph-H), 7.42 (d, $J=7.9 \mathrm{~Hz}, 1 \mathrm{H}, \mathrm{Ph}-\mathrm{H})$, 7.36 (dd, $J=8.9,0.9 \mathrm{~Hz}, 2 \mathrm{H}, \mathrm{Ph}-\mathrm{H}), 7.28$ (td, $J=7.5,0.9$ $\mathrm{Hz}, 1 \mathrm{H}, \mathrm{Ph}-\mathrm{H}), 6.38$ (s, 1H, Py-H), 3.79 (s, 6H, $\mathrm{OCH}_{3}$ ), $3.12\left(\mathrm{~s}, 3 \mathrm{H}, \mathrm{NCH}_{3}\right) ;{ }^{13} \mathrm{C}$ NMR $\left(\mathrm{CDCl}_{3}, 101 \mathrm{MHz}\right) \delta$ : $190.47,171.52,170.36,161.89,156.10,151.17$ (q, $J=1.7$ $\mathrm{Hz}), 134.01,131.63,130.84,130.23,125.81,123.41$, 120.20 (q, $J=258.5 \mathrm{~Hz}), 119.90$ (d, $J=0.6 \mathrm{~Hz}), 112.35$, 91.16, 54.31, 35.37. HRMS calcd for $\mathrm{C}_{22} \mathrm{H}_{18} \mathrm{~F}_{3} \mathrm{~N}_{3} \mathrm{NaO}_{6}$ $[\mathrm{M}+\mathrm{Na}]^{+}$500.1040, found 500.1034.

4-\{[2-(4,6-二甲氧基嘧啶基-2-甲酰基)苯氧基] (甲 基)胺基甲酰基 $\}$ 苯甲酸甲酯(4AHq): m.p. $126 \sim 128{ }^{\circ} \mathrm{C}$; HPLC: $97.8 \%$; ${ }^{1} \mathrm{H}$ NMR (DMSO- $\left.d_{6}, 300 \mathrm{MHz}\right) \delta: 7.93 \sim$ 7.84 (m, 2H, Ph-H), $7.72 \sim 7.64$ (m, 2H, Ph-H), $7.53 \sim 7.47$ (m, 2H, Ph-H), $7.43 \sim 7.37$ (m, 1H, Ph-H), 7.26 (t, $J=7.5$ $\mathrm{Hz}, 1 \mathrm{H}, \mathrm{Ph}-\mathrm{H}), 6.36$ (s, 1H, Py-H), 3.85 (s, 3H, $\mathrm{COOCH}_{3}$ ),

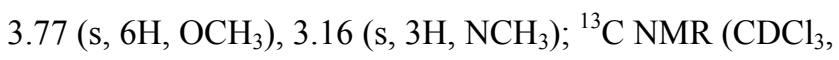
$101 \mathrm{MHz}) \delta$ : 190.49, 171.72, 171.10, 166.06, 161.82, $156.03,136.84,133.90,132.22,131.50,129.15,127.78$, 125.77, 123.32, 112.17, 91.27, 54.31, 52.23, 35.26. HRMS calcd for $\mathrm{C}_{23} \mathrm{H}_{21} \mathrm{ClN}_{3} \mathrm{NaO}_{7}[\mathrm{M}+\mathrm{Na}]^{+}$474.1272, found 474.1267.

3-氯- $N$-[2-(4,6-二甲氧基嘧啶基-2-甲酰基)-3-氟苯 氧基]- $\mathrm{N}$-甲基苯甲酰胺 (4AFa): m.p. $112 \sim 114{ }^{\circ} \mathrm{C}$; HPLC: $89.1 \%$; ${ }^{1} \mathrm{H}$ NMR (DMSO- $\left.d_{6}, 300 \mathrm{MHz}\right) \delta: 7.59$ (dd, $J=15.0,8.4 \mathrm{~Hz}, 1 \mathrm{H}, \mathrm{Ph}-\mathrm{H}), 7.49 \sim 7.41(\mathrm{~m}, 2 \mathrm{H}$, Ph-H), $7.38 \sim 7.27$ (m, 2H, Ph-H), 7.22 (d, J=8.5 Hz, 1H, Ph-H), 7.08 (t, $J=8.7$ Hz, 1H, Ph-H), 6.46 (s, 1H, Py-H), $3.78\left(\mathrm{~s}, 6 \mathrm{H}, \mathrm{OCH}_{3}\right), 3.19$ (s, $\left.3 \mathrm{H}, \mathrm{NCH}_{3}\right) ;{ }^{13} \mathrm{C} \mathrm{NMR}\left(\mathrm{CDCl}_{3}\right.$, $101 \mathrm{MHz}) \delta: 187.69,171.65,170.39,160.58(\mathrm{~d}, J=250.6$ Hz), 158.89, 155.97 (d, $J=6.9 \mathrm{~Hz}), 134.32,134.02,132.02$ (d, $J=10.0 \mathrm{~Hz}), 131.17,129.15,128.26,126.01,116.41$ (d, $J=21.7 \mathrm{~Hz}), 110.48$ (d, $J=21.8 \mathrm{~Hz}), 107.83$ (d, $J=3.0$ $\mathrm{Hz}$ ), 92.83, 54.28, 36.06. HRMS calcd for $\mathrm{C}_{21} \mathrm{H}_{17} \mathrm{ClF}-$ $\mathrm{N}_{3} \mathrm{NaO}_{5}[\mathrm{M}+\mathrm{Na}]^{+}$468.0733, found 468.0734.

4-氯- $N$-[2-(4,6-二甲氧基嘧啶基-2-甲酰基)-3-氟苯 氧基 ]- $N$ - 甲基苯甲酰胺 $(\mathbf{4 A F b})$ : m.p. $116 \sim 118{ }^{\circ} \mathrm{C}$; HPLC: $96.3 \%$; ${ }^{1} \mathrm{H}$ NMR (DMSO- $\left.d_{6}, 300 \mathrm{MHz}\right) \delta: 7.66 \sim$ $7.56(\mathrm{~m}, 1 \mathrm{H}, \mathrm{Ph}-\mathrm{H}), 7.45 \sim 7.33$ (m, 4H, Ph-H), 7.23 (d, $J=8.5 \mathrm{~Hz}, 1 \mathrm{H}, \mathrm{Ph}-\mathrm{H}), 7.10(\mathrm{t}, J=8.8 \mathrm{~Hz}, 1 \mathrm{H}, \mathrm{Ph}-\mathrm{H}), 6.48$ (s, $1 \mathrm{H}, \mathrm{Py}-\mathrm{H}), 3.80\left(\mathrm{~s}, 6 \mathrm{H}, \mathrm{OCH}_{3}\right), 3.21\left(\mathrm{~s}, 3 \mathrm{H}, \mathrm{NCH}_{3}\right) ;{ }^{13} \mathrm{C}$ NMR $\left(\mathrm{CDCl}_{3}, 100 \mathrm{MHz}\right) \delta$ : 187.75, 171.67, 170.77, $160.60(\mathrm{~d}, J=250.8 \mathrm{~Hz}), 158.96,155.97(\mathrm{~d}, J=7.0 \mathrm{~Hz})$, $137.43,132.05(\mathrm{~d}, J=10.0 \mathrm{~Hz}), 130.86,129.67,128.18$, 116.37 (d, $J=21.6 \mathrm{~Hz}), 110.48$ (d, $J=21.8 \mathrm{~Hz}), 107.64$ (d, $J=3.1 \mathrm{~Hz}), 92.72,54.29,35.92$. HRMS calcd for $\mathrm{C}_{21} \mathrm{H}_{17^{-}}$ $\mathrm{ClFN}_{3} \mathrm{NaO}_{5}[\mathrm{M}+\mathrm{Na}]^{+}$468.0733, found 468.0755.

2-氯- $N$-[2-(4,6-二甲氧基嘧啶基-2-甲酰基)-3-氟苯 氧基]- $N$-甲基苯甲酰胺 $(\mathbf{4 A F c}):$ m.p. $99 \sim 101{ }^{\circ} \mathrm{C}$; HPLC: 99.2\%; ${ }^{1} \mathrm{H}$ NMR (DMSO- $\left.d_{6}, 300 \mathrm{MHz}\right) \delta: 7.56$ (dd, $J=$ 15.0, $8.4 \mathrm{~Hz}, 1 \mathrm{H}, \mathrm{Ph}-\mathrm{H}), 7.39 \sim 7.13$ (m, 5H, Ph-H), 7.06 (t, $J=8.8 \mathrm{~Hz}, 1 \mathrm{H}, \mathrm{Ph}-\mathrm{H}), 6.48$ (s, 1H, Py-H), 3.75 (s, 6H, $\left.\mathrm{OCH}_{3}\right), 3.20$ (s, $\left.3 \mathrm{H}, \mathrm{NCH}_{3}\right) ;{ }^{13} \mathrm{C} \mathrm{NMR}\left(\mathrm{CDCl}_{3}, 101 \mathrm{MHz}\right)$ $\delta: 187.78,171.57,169.76,160.30(\mathrm{~d}, J=250.6 \mathrm{~Hz})$, 158.81, 155.86 (d, $J=6.6 \mathrm{~Hz}), 133.78,131.73$ (d, $J=9.9$ $\mathrm{Hz}), 130.60,129.24,127.44,126.17,116.43$ (d, $J=21.2$ $\mathrm{Hz}), 110.44$ (d, $J=21.7 \mathrm{~Hz}), 107.63,92.86,54.24,35.21$. HRMS calcd for $\mathrm{C}_{21} \mathrm{H}_{17} \mathrm{ClFN}_{3} \mathrm{NaO}_{5}[\mathrm{M}+\mathrm{Na}]^{+} 468.0733$, found 468.0755 .

$N$-[2-(4,6-二甲氧基嘧啶基-2-甲酰基)-3-氟苯氧 基]- $N, 2$-二甲基苯甲酰胺 (4AFd): m.p. $120 \sim 122{ }^{\circ} \mathrm{C}$; HPLC: $96.4 \%$; ${ }^{1} \mathrm{H}$ NMR (DMSO- $\left.d_{6}, 300 \mathrm{MHz}\right) \delta: 7.56(\mathrm{td}$, $J=8.5,6.5 \mathrm{~Hz}, 1 \mathrm{H}, \mathrm{Ph}-\mathrm{H}), 7.20 \sim 6.88(\mathrm{~m}, 6 \mathrm{H}, \mathrm{Ph}-\mathrm{H}), 6.48$ (s, 1H, Py-H), 3.76 (s, 6H, $\left.\mathrm{OCH}_{3}\right), 3.18$ (s, 3H, $\mathrm{NCH}_{3}$ ), $2.04\left(\mathrm{~s}, 3 \mathrm{H}, \mathrm{Ph}-\mathrm{CH}_{3}\right) ;{ }^{13} \mathrm{C}$ NMR $\left(\mathrm{CDCl}_{3}, 101 \mathrm{MHz}\right) \delta$ : $187.93,172.54,171.52,160.35$ (d, $J=250.4 \mathrm{~Hz}), 158.69$, $156.10(\mathrm{~d}, J=7.0 \mathrm{~Hz}), 134.79,133.78,131.69$ (d, $J=9.9$ Hz), 129.85, 129.44, 125.83, 124.94, 116.25 (d, $J=21.7$ $\mathrm{Hz}), 110.18$ (d, $J=21.7 \mathrm{~Hz}), 107.46,92.90,54.23,35.27$, 19.05. HRMS calcd for $\mathrm{C}_{22} \mathrm{H}_{20} \mathrm{FN}_{3} \mathrm{NaO}_{5}[\mathrm{M}+\mathrm{Na}]^{+}$ 448.1279, found 448.1291.

$N$-[2-(4,6-二甲氧基嘧啶基-2-甲酰基)-3-氟苯氧 基]- $N, 3$-二甲基苯甲酰胺(4AFe): oil; HPLC： $89.6 \% ;{ }^{1} \mathrm{H}$ NMR (DMSO- $\left.d_{6}, 400 \mathrm{MHz}\right) \delta: 7.65 \sim 7.55(\mathrm{~m}, 1 \mathrm{H}, \mathrm{Ph}-\mathrm{H})$, $7.27 \sim 7.14$ (m, 5H, Ph-H), 7.08 (t, $J=8.8 \mathrm{~Hz}, 1 \mathrm{H}, \mathrm{Ph}-\mathrm{H})$, 6.47 (d, $J=0.5 \mathrm{~Hz}, 1 \mathrm{H}, \mathrm{Py}-\mathrm{H}), 3.80$ (s, 6H, $\left.\mathrm{OCH}_{3}\right), 3.18$ (s, $\left.3 \mathrm{H}, \mathrm{NCH}_{3}\right), 2.23$ (s, 3H, Ph- $\left.\mathrm{CH}_{3}\right) ;{ }^{13} \mathrm{C}$ NMR $\left(\mathrm{CDCl}_{3}, 101\right.$ MHz) $\delta: 187.82,171.98,171.44,160.08(\mathrm{~d}, J=247.5 \mathrm{~Hz})$, $159.17,156.48$ (d, $J=6.8 \mathrm{~Hz}), 137.93,133.40$ (d, $J=10.2$ $\mathrm{Hz}), 133.14,132.19,128.52,128.31,124.98,115.74(\mathrm{~d}$, $J=21.2 \mathrm{~Hz}), 110.52(\mathrm{~d}, J=21.4 \mathrm{~Hz}), 109.11$ (d, $J=2.7$ $\mathrm{Hz}), 92.50$, 54.83, 37.40, 21.10. HRMS calcd for $\mathrm{C}_{22} \mathrm{H}_{20} \mathrm{FN}_{3} \mathrm{NaO}_{5}[\mathrm{M}+\mathrm{Na}]^{+}$448.1279, found 448.1292. 
2,3-二氯- $N$-[2-(4,6-二甲氧基嘧啶基-2-甲酰基)-3-氟 苯氧基]- $N$-甲基苯甲酰胺(4AFf): m.p. $104 \sim 106{ }^{\circ} \mathrm{C}$; HPLC: $84.6 \%$; ${ }^{1} \mathrm{H}$ NMR (DMSO- $\left.d_{6}, 300 \mathrm{MHz}\right) \delta: 7.65 \sim$ 7.42 (m, 2H, Ph-H), $7.34 \sim 7.15(\mathrm{~m}, 3 \mathrm{H}, \mathrm{Ph}-\mathrm{H}), 7.08$ (t, $J=8.6 \mathrm{~Hz}, 1 \mathrm{H}, \mathrm{Ph}-\mathrm{H}), 6.47$ (s, 1H, Py-H), 3.76 (s, 6H, $\left.\mathrm{OCH}_{3}\right), 3.29$ (s, 3H, $\left.\mathrm{NCH}_{3}\right) ;{ }^{13} \mathrm{C} \mathrm{NMR}\left(\mathrm{CDCl}_{3}, 101 \mathrm{MHz}\right)$ $\delta: 187.59,171.53,169.06,160.34(\mathrm{~d}, J=250.8 \mathrm{~Hz})$, 158.62, 155.67 (d, $J=6.6 \mathrm{~Hz}), 135.87,133.20,131.75$ (d, $J=9.9 \mathrm{~Hz}), 131.17,129.10,126.99,125.33,116.41$ (d, $J=$ $26.5 \mathrm{~Hz}), 110.57$ (d, $J=21.7 \mathrm{~Hz}), 107.37,93.03,54.24$, 35.05. HRMS calcd for $\mathrm{C}_{21} \mathrm{H}_{16} \mathrm{Cl}_{2} \mathrm{FN}_{3} \mathrm{NaO}_{5}[\mathrm{M}+\mathrm{Na}]^{+}$ 502.0343, found 502.0353.

2,5-二氯- $N$-[2-(4,6-二甲氧基嘧啶基-2-甲酰基)-3-氟 苯氧基]- $N$-甲基苯甲酰胺(4AFg): m.p. $118 \sim 119{ }^{\circ} \mathrm{C}$; HPLC: $91.6 \%$; ${ }^{1} \mathrm{H}$ NMR (DMSO- $\left.d_{6}, 300 \mathrm{MHz}\right) \delta: 7.67 \sim$ $7.46(\mathrm{~m}, 2 \mathrm{H}, \mathrm{Ph}-\mathrm{H}), 7.38 \sim 7.20(\mathrm{~m}, 3 \mathrm{H}, \mathrm{Ph}-\mathrm{H}), 7.09$ (t, $J=8.7 \mathrm{~Hz}, 1 \mathrm{H}, \mathrm{Ph}-\mathrm{H}), 6.49$ (s, 1H, Py-H), 3.77 (s, 6H, $\left.\mathrm{OCH}_{3}\right), 3.27$ (s, 3H, $\left.\mathrm{NCH}_{3}\right) ;{ }^{13} \mathrm{C} \mathrm{NMR}\left(\mathrm{CDCl}_{3}, 101 \mathrm{MHz}\right)$ $\delta$ : 187.65, 171.56, 168.48, $160.31(\mathrm{~d}, J=250.7 \mathrm{~Hz})$, $158.65,155.58$ (d, $J=6.3 \mathrm{~Hz}), 135.18,132.31,131.75$ (d, $J=9.9 \mathrm{~Hz}), 130.58,130.38,128.95,127.30,116.43$ (d, $J=$ $22.6 \mathrm{~Hz}), 110.62$ (d, $J=21.7 \mathrm{~Hz}), 107.37,93.00,54.25$, 35.10. HRMS calcd for $\mathrm{C}_{21} \mathrm{H}_{16} \mathrm{Cl}_{2} \mathrm{FN}_{3} \mathrm{NaO}_{5}[\mathrm{M}+\mathrm{Na}]^{+}$ 502.0343, found 502.0350.

2,6-二氯- $N$-[2-(4,6-二甲氧基嘧啶基-2-甲酰基)-3-氟 苯氧基]- $N$-甲基苯甲酰胺(4AFh): m.p. $133 \sim 134{ }^{\circ} \mathrm{C}$; HPLC: $97.5 \%$; ${ }^{1} \mathrm{H}$ NMR (DMSO- $\left.d_{6}, 300 \mathrm{MHz}\right) \delta: 7.64 \sim$ 7.53 (m, 1H, Ph-H), 7.25 (s, 3H, Ph-H), 7.22 (d, $J=8.5$ $\mathrm{Hz}, 1 \mathrm{H}, \mathrm{Ph}-\mathrm{H}), 7.09$ (t, J=8.7 Hz, 1H, Ph-H), 6.48 (s, 1H, Py-H), 3.75 (s, 6H, $\mathrm{OCH}_{3}$ ), 3.34 (s, 3H, $\left.\mathrm{NCH}_{3}\right) ;{ }^{13} \mathrm{C} \mathrm{NMR}$ $\left(\mathrm{CDCl}_{3}, 101 \mathrm{MHz}\right) \delta: 187.58,171.49,167.73,160.17$ (d, $J=251.0 \mathrm{~Hz}$ ), 158.79, 155.50 (d, $J=6.9 \mathrm{~Hz}), 133.46$, $131.74(\mathrm{~d}, J=9.9 \mathrm{~Hz}), 131.59,130.55,127.33,116.44$ (d, $J=21.4 \mathrm{~Hz}), 110.65(\mathrm{~d}, J=21.6 \mathrm{~Hz}), 107.91(\mathrm{~d}, J=3.0$ $\mathrm{Hz}$ ), 92.86, 54.20, 34.98. HRMS calcd for $\mathrm{C}_{21} \mathrm{H}_{16} \mathrm{Cl}_{2} \mathrm{~F}-$ $\mathrm{N}_{3} \mathrm{NaO}_{5}[\mathrm{M}+\mathrm{Na}]^{+}$502.0343, found 502.0351.

$N$-[2-(4,6-二甲氧基嘧啶基-2-甲酰基)-3-氟苯氧 基]- $N$-甲基噻吩-2-甲酰胺(4AFi): m.p. $135 \sim 136{ }^{\circ} \mathrm{C}$; HPLC: $98.0 \%$; ${ }^{1} \mathrm{H}$ NMR (DMSO- $\left.d_{6}, 300 \mathrm{MHz}\right) \delta$ : $7.86(\mathrm{~d}$, $J=4.0 \mathrm{~Hz}, 1 \mathrm{H}, \mathrm{Ar}-\mathrm{H}), 7.66 \sim 7.56(\mathrm{~m}, 2 \mathrm{H}, \mathrm{Ar}-\mathrm{H}), 7.22 \sim$ $7.12(\mathrm{~m}, 2 \mathrm{H}, \mathrm{Ar}-\mathrm{H}), 7.11 \sim 7.05(\mathrm{~m}, 1 \mathrm{H}, \mathrm{Ar}-\mathrm{H}), 6.47$ (s, $1 \mathrm{H}, \mathrm{Py}-\mathrm{H}), 3.82$ (s, 6H, $\left.\mathrm{OCH}_{3}\right), 3.17\left(\mathrm{~s}, 3 \mathrm{H}, \mathrm{NCH}_{3}\right) ;{ }^{13} \mathrm{C}$ NMR $\left(\mathrm{CDCl}_{3}, 101 \mathrm{MHz}\right) \delta: 187.70,171.75,164.37$, 160.65 (d, $J=250.8 \mathrm{~Hz}), 159.22,155.83$ (d, $J=6.8 \mathrm{~Hz})$, 134.21, 133.41, 132.76, 132.14 (d, $J=9.9 \mathrm{~Hz}), 127.26$, $116.88(\mathrm{~d}, J=21.6 \mathrm{~Hz}), 110.84(\mathrm{~d}, J=21.7 \mathrm{~Hz}), 108.60$ (d, $J=3.1 \mathrm{~Hz})$, 92.73, 54.37, 35.54. HRMS calcd for $\mathrm{C}_{19} \mathrm{H}_{16} \mathrm{FN}_{3} \mathrm{NaO}_{5} \mathrm{~S}[\mathrm{M}+\mathrm{Na}]^{+}$440.0687, found 440.0702.

$N$-[2-(4,6-二甲氧基嘧啶基-2-甲酰基)-3-氟苯氧 基]- $N$-甲基呋喃-2-甲酰胺(4AFj): m.p. $112 \sim 113{ }^{\circ} \mathrm{C}$; HPLC: $99.2 \%$; ${ }^{1} \mathrm{H}$ NMR (DMSO- $\left.d_{6}, 300 \mathrm{MHz}\right) \delta: 7.87$ (s, $1 \mathrm{H}, \mathrm{Ar}-\mathrm{H}), 7.65 \sim 7.54(\mathrm{~m}, 1 \mathrm{H}, \mathrm{Ar}-\mathrm{H}), 7.20 \sim 7.09(\mathrm{~m}, 2 \mathrm{H}$, Ar-H), 6.96 (d, J=3.5 Hz, 1H, Ar-H), 6.60 6.54 (m, 1H, Ar-H), 6.48 (s, 1H, Py-H), 3.83 (s, 6H, $\left.\mathrm{OCH}_{3}\right), 3.16$ (s, 3H, $\left.\mathrm{NCH}_{3}\right) ;{ }^{13} \mathrm{C} \mathrm{NMR}\left(\mathrm{CDCl}_{3}, 101 \mathrm{MHz}\right) \delta: 187.87,171.78$, $160.94,160.63$ (d, $J=250.6 \mathrm{~Hz}), 159.16,156.11$ (d, $J=6.8$ $\mathrm{Hz}), 145.92,144.55,132.22(\mathrm{~d}, J=10.0 \mathrm{~Hz}), 118.85$, $116.51(\mathrm{~d}, J=21.5 \mathrm{~Hz}), 111.77,110.58(\mathrm{~d}, J=21.7 \mathrm{~Hz})$, 108.22 (d, $J=3.1 \mathrm{~Hz}$ ), 92.73, 54.35, 35.44. HRMS calcd for $\mathrm{C}_{19} \mathrm{H}_{16} \mathrm{FN}_{3} \mathrm{NaO}_{6}[\mathrm{M}+\mathrm{H}]^{+}$402.1096, found 402.1096.

5-氯- $N$-[2-(4,6-二甲氧基嘧啶基-2-甲酰基)-3-氟苯 氧基]- $N, 1,3$-三甲基- $1 H$-吡唑-4-甲酰胺 (4AFk)：m.p. 116 118 ${ }^{\circ} \mathrm{C}$; HPLC: 94.7\%; ${ }^{1} \mathrm{H}$ NMR (DMSO- $d_{6}, 300$ $\mathrm{MHz}) \delta: 7.59(\mathrm{dd}, J=15.1,8.3 \mathrm{~Hz}, 1 \mathrm{H}, \mathrm{Ph}-\mathrm{H}), 7.15 \sim 7.02$ (m, 1H, Ph-H), 6.96 (d, J=8.3Hz, 1H, Ph-H), 6.53 (s, 1H, Py-H), 3.81 (s, 6H, $\left.\mathrm{OCH}_{3}\right), 3.62\left(\mathrm{~s}, 3 \mathrm{H}\right.$, pyrazole-1- $\left.\mathrm{CH}_{3}\right)$, 3.22 (s, 3H, O-N-CH$), 2.03$ (s, 3H, pyrazole-3- $\left.\mathrm{CH}_{3}\right) ;{ }^{13} \mathrm{C}$ NMR $\left(\mathrm{CDCl}_{3}, 101 \mathrm{MHz}\right) \delta: 187.84,171.68,164.77$, $160.40(\mathrm{~d}, J=250.8 \mathrm{~Hz}), 159.08,156.34$ (d, $J=6.9 \mathrm{~Hz})$, $148.11,131.77$ (d, $J=9.9 \mathrm{~Hz}), 127.48,116.69$ (d, $J=21.3$ $\mathrm{Hz}), 110.82,110.47$ (d, $J=21.7 \mathrm{~Hz}), 107.45$ (d, $J=3.1$ $\mathrm{Hz})$, 92.83, 54.30, 35.92, 35.16, 13.08. HRMS calcd for $\mathrm{C}_{20} \mathrm{H}_{19} \mathrm{ClFN}_{5} \mathrm{NaO}_{5}[\mathrm{M}+\mathrm{Na}]^{+}$486.0951, found 486.0954.

2,2,3,3-四氯- $N$-[2-(4,6-二甲氧基嘧啶基-2-甲酰 基)-3-氟苯氧基]- $N, 1$-二甲基环丙基-1-甲酰胺(4AFI): m.p. $150 \sim 151{ }^{\circ} \mathrm{C}$; HPLC: 98.4\%; ${ }^{1} \mathrm{H}$ NMR (DMSO- $d_{6}$, $300 \mathrm{MHz}) \delta: 7.65$ (dd, $J=15.1,8.3 \mathrm{~Hz}, 1 \mathrm{H}, \mathrm{Ph}-\mathrm{H}), 7.27 \sim$ 7.10 (m, 2H, Ph-H), 6.49 (s, 1H, Py-H), 3.83 (s, 6H, $\left.\mathrm{OCH}_{3}\right), 3.12\left(\mathrm{~s}, 3 \mathrm{H}, \mathrm{NCH}_{3}\right), 1.92(\mathrm{~d}, J=7.8 \mathrm{~Hz}, 1 \mathrm{H}$, cyclo-Pr-H), 1.59 (d, $J=7.7 \mathrm{~Hz}, 1 \mathrm{H}$, cyclo-Pr-H), 1.29 (s, $3 \mathrm{H}$, cyclo-Pr- $\left.\mathrm{CH}_{3}\right) ;{ }^{13} \mathrm{C} \mathrm{NMR}\left(\mathrm{CDCl}_{3}, 101 \mathrm{MHz}\right) \delta$ : $187.86,175.89,171.77,160.63$ (d, $J=250.6 \mathrm{~Hz}), 159.39$, 157.43 (d, $J=6.8 \mathrm{~Hz}), 131.82$ (d, $J=10.1 \mathrm{~Hz}), 116.04$ (d, $J=21.2 \mathrm{~Hz}), 109.92$ (d, $J=21.8 \mathrm{~Hz}), 108.00$ (d, $J=3.1$ $\mathrm{Hz})$, 92.61, 54.32, 34.79, 33.36, 30.19, 23.32, 16.81. HRMS calcd for $\mathrm{C}_{19} \mathrm{H}_{18} \mathrm{Cl}_{2} \mathrm{FN}_{3} \mathrm{NaO}_{5}[\mathrm{M}+\mathrm{Na}]^{+} 480.0500$, found 480.0500 .

$N$-[2-(4,6-二甲氧基嘧啶基-2-甲酰基)-3-氟苯氧 基]- $N, 2,2,3,3$ - 四甲基环丙基-1- 甲酰胺(4AFm): m.p. 116 $117{ }^{\circ} \mathrm{C}$; HPLC: 99.3\%; ${ }^{1} \mathrm{H}$ NMR (DMSO- $d_{6}, 300$ 
MHz) $\delta$ : $7.61(\mathrm{dd}, J=15.0,8.4 \mathrm{~Hz}, 1 \mathrm{H}, \mathrm{Ph}-\mathrm{H}), 7.15 \sim 6.96$ (m, 2H, Ph-H), 6.53 (s, 1H, Py-H), $3.85\left(\mathrm{~s}, 6 \mathrm{H}, \mathrm{OCH}_{3}\right)$, $2.98\left(\mathrm{~s}, 3 \mathrm{H}, \mathrm{NCH}_{3}\right), 1.18$ (s, 1H, cyclo-Pr-H), 1.08 (s, 6H, cyclo-Pr- $\mathrm{CH}_{3}$ ), 0.93 (s, 6H, cyclo- $\left.\mathrm{Pr}-\mathrm{CH}_{3}\right) ;{ }^{13} \mathrm{C}$ NMR $\left(\mathrm{CDCl}_{3}, 101 \mathrm{MHz}\right) \delta: 187.62,171.75,160.82(\mathrm{~d}, J=251.4$ $\mathrm{Hz}), 159.14,155.94$ (d, $J=7.0 \mathrm{~Hz}), 132.00$ (d, $J=10.0$ $\mathrm{Hz}), 116.80(\mathrm{~d}, J=21.1 \mathrm{~Hz}), 110.62(\mathrm{~d}, J=21.7 \mathrm{~Hz})$, 107.93, 92.76, 62.07, 54.32, 36.29, 35.80, 31.10, 18.63 . HRMS calcd for $\mathrm{C}_{22} \mathrm{H}_{26} \mathrm{FN}_{3} \mathrm{NaO}_{5} \quad[\mathrm{M}+\mathrm{Na}]^{+}$454.1749, found 454.1771 .

$N$-[2-(4,6-二甲氧基-5-甲基嘧啶基-2-甲酰基)苯氧 基]- $N, 2$-二甲基苯甲酰胺(4BHa): m.p. $121 \sim 123{ }^{\circ} \mathrm{C}$; HPLC: $98.8 \%$; ${ }^{1} \mathrm{H}$ NMR (DMSO- $\left.d_{6}, 300 \mathrm{MHz}\right) \delta: 7.69 \sim$ 7.56 (m, 2H, Ph-H), 7.35 (d, J=8.1 Hz, 1H, Ph-H), 7.26 7.01 (m, 5H, Ph-H), 3.78 (s, 6H, $\left.\mathrm{OCH}_{3}\right), 3.11(\mathrm{~s}, 3 \mathrm{H}$, $\mathrm{NCH}_{3}$ ), $2.09 \sim 1.96\left(\mathrm{~d}, 6 \mathrm{H}, \mathrm{Ph}-\mathrm{CH}_{3}\right.$ and $\left.\mathrm{Py} .-\mathrm{CH}_{3}\right) ;{ }^{13} \mathrm{C}$ NMR $\left(\mathrm{CDCl}_{3}, 101 \mathrm{MHz}\right) \delta$ : 191.63, 172.38, 168.23, $157.66,155.84,134.78,133.95,132.84,130.73,129.98$, $129.37,126.95,125.77,125.09,122.98,112.25,102.66$, 54.30, 34.70, 18.96, 7.61. HRMS calcd for $\mathrm{C}_{23} \mathrm{H}_{23} \mathrm{~N}_{3} \mathrm{NaO}_{5}$ $[\mathrm{M}+\mathrm{Na}]^{+} 444.1530$, found 444.1550 .

$N$-[2-(4,6-二乙氧基嘧啶基-2-甲酰基)苯氧基]- $N, 2$ 二甲基苯甲酰胺 $(\mathbf{4 C H a}):$ m.p. $120 \sim 121{ }^{\circ} \mathrm{C}$; HPLC: 99.1\%; ${ }^{1} \mathrm{H}$ NMR (DMSO- $\left.d_{6}, 300 \mathrm{MHz}\right) \delta: 7.65$ (t, $J=8.5$ $\mathrm{Hz}, 2 \mathrm{H}, \mathrm{Ph}-\mathrm{H}$ ), 7.35 (d, J=8.2 Hz, 1H, Ph-H), $7.28 \sim 7.00$ (m, 5H, Ph-H), 6.36 (s, 1H, Py-H), 4.21 (q, J=7.0 Hz, 4H, $\mathrm{CH}_{2} \mathrm{CH}_{3}$ ), 3.09 (s, 3H, NCH$)_{3}$, 2.07 (s, 3H, Ph- $\mathrm{CH}_{3}$ ), 1.27 (t, $\left.J=7.0 \mathrm{~Hz}, 6 \mathrm{H}, \mathrm{CH}_{2} \mathrm{CH}_{3}\right) ;{ }^{13} \mathrm{C} \mathrm{NMR}\left(\mathrm{CDCl}_{3}, 101 \mathrm{MHz}\right)$ $\delta: 190.98,172.37,171.11,161.76,156.24,134.91,133.79$, $133.49,131.13,130.13,129.46,126.01,125.82,125.13$, 123.12, 112.36, 91.40, 63.00, 34.72, 19.10, 14.35. HRMS calcd for $\mathrm{C}_{24} \mathrm{H}_{25} \mathrm{~N}_{3} \mathrm{NaO}_{5}[\mathrm{M}+\mathrm{Na}]^{+}$458.1686, found 458.1697 .

2,3-二氯- $N$-[2-(4,6-二乙氧基嘧啶基-2-甲酰基)-3-氟 苯氧基]- $N$-甲基苯甲酰胺 $(\mathbf{4 C F a})$ : m.p. $121 \sim 122{ }^{\circ} \mathrm{C}$; HPLC: $98.5 \%$; ${ }^{1} \mathrm{H}$ NMR (DMSO- $\left.d_{6}, 300 \mathrm{MHz}\right) \delta: 7.79 \sim$ 7.14 (m, 5H, Ph-H), 7.07 (t, $J=8.6 \mathrm{~Hz}, 1 \mathrm{H}, \mathrm{Ph}-\mathrm{H}), 6.38$ (s, $1 \mathrm{H}, \mathrm{Py}-\mathrm{H}$ ), 4.16 (d, $\left.J=6.9 \mathrm{~Hz}, 4 \mathrm{H}, \mathrm{CH}_{2} \mathrm{CH}_{3}\right), 3.29$ (s, $3 \mathrm{H}$, $\left.\mathrm{NCH}_{3}\right), 1.24$ (t, $\left.J=7.0 \mathrm{~Hz}, 6 \mathrm{H}, \mathrm{CH}_{2} \mathrm{CH}_{3}\right) ;{ }^{13} \mathrm{C} \mathrm{NMR}$ $\left(\mathrm{CDCl}_{3}, 101 \mathrm{MHz}\right) \delta$ : 187.79, 171.20, 169.06, $160.29(\mathrm{~d}$, $J=250.4 \mathrm{~Hz}), 158.59,155.59(\mathrm{~d}, J=5.0 \mathrm{~Hz}), 135.80$, 133.18, $131.68(\mathrm{~d}, J=9.8 \mathrm{~Hz}), 131.10,129.11,126.93$, 125.36, 116.49 (d, J=26.5 Hz), $110.52(\mathrm{~d}, J=21.7 \mathrm{~Hz})$, 107.30, 93.20, 63.03, 34.97, 14.27. HRMS calcd for $\mathrm{C}_{23} \mathrm{H}_{20} \mathrm{Cl}_{2} \mathrm{FN}_{3} \mathrm{NaO}_{5}[\mathrm{M}+\mathrm{H}]^{+}$508.0837, found 508.0829.
2,6-二氯- $N$-[2-(4,6-二乙氧基嘧啶基-2-甲酰基)-3-氟 苯氧基]- $N$-甲基苯甲酰胺 (4CFb): m.p. $125 \sim 126{ }^{\circ} \mathrm{C}$; HPLC: $99.4 \%$; ${ }^{1} \mathrm{H}$ NMR (DMSO- $\left.d_{6}, 300 \mathrm{MHz}\right) \delta: 7.59$ (dd, $J=14.9,8.2 \mathrm{~Hz}, 2 \mathrm{H}, \mathrm{Ph}-\mathrm{H}), 7.31 \sim 7.00(\mathrm{~m}, 4 \mathrm{H}$, $\mathrm{Ph}-\mathrm{H}), 6.39$ (s, 1H, Py-H), $4.35 \sim 4.03$ (m, 4H, $\mathrm{CH}_{2} \mathrm{CH}_{3}$ ), $3.35\left(\mathrm{~s}, 2 \mathrm{H}, \mathrm{NCH}_{3}\right), 3.06\left(\mathrm{~s}, 1 \mathrm{H}, \mathrm{NCH}_{3}\right), 1.25$ (t, $J=7.0$ $\left.\mathrm{Hz}, 6 \mathrm{H}, \mathrm{CH}_{2} \mathrm{CH}_{3}\right) ;{ }^{13} \mathrm{C} \mathrm{NMR}\left(\mathrm{CDCl}_{3}, 101 \mathrm{MHz}\right) \delta: 187.77$, $171.17,167.80,160.12(\mathrm{~d}, J=250.6 \mathrm{~Hz}), 158.73,155.43$ (d, $J=7.1 \mathrm{~Hz}), 133.43,131.65(\mathrm{~d}, J=4.9 \mathrm{~Hz}), 131.57$, $130.50,127.30,116.54$ (d, $J=21.8 \mathrm{~Hz}), 110.56$ (d, $J=21.6$ Hz), 107.86 (d, $J=3.1 \mathrm{~Hz}), 93.01,62.99,35.02,14.28$. HRMS calcd for $\mathrm{C}_{23} \mathrm{H}_{20} \mathrm{Cl}_{2} \mathrm{FN}_{3} \mathrm{NaO}_{5}[\mathrm{M}+\mathrm{Na}]^{+}$530.0656, found 530.0681 .

3.2.2 $N$-\{2-[(4,6-二甲氧基嘧啶-2-基)(羟基)甲基]苯 氧基\}- $N$-甲基 $\mathrm{R}^{1}$ 取代甲酰胺 $\mathbf{5 A H}$ 的合成

将化合物 4AH (1 mmol) 溶于 $30 \mathrm{~mL}$ 甲醇中, 降温至 $0{ }^{\circ} \mathrm{C}$ 后, 缓慢加入嗍氢化钠 $(38 \mathrm{mg}, 1 \mathrm{mmol})$. 反应 $2 \mathrm{~h}$ 后, 将反应液倒入水中, 乙酸乙酯 $(30 \mathrm{~mL} \times 3)$ 萃取分液, 有 机相经水洗、干燥、旋干后得粗品, 粗品经乙酸乙酯/ 石油醚柱层析后得相应的化合物 $\mathbf{5 A H}$.

2,6-二氯- $N$ - $\{2-[(4,6-$ 二甲氧基嘧啶-2-基)(羟基)甲 基]苯氧基 $\}-N$-甲基苯甲酰胺(5AHa): m.p. $116 \sim 118{ }^{\circ} \mathrm{C}$; HPLC: $96.8 \% ;{ }^{1} \mathrm{H}$ NMR (DMSO- $\left.d_{6}, 300 \mathrm{MHz}\right) \delta: 7.69 \sim$ 7.47 (m, 2H, Ph-H), 7.42 7.02 (m, 5H, Ph-H), 6.05 (s, 1H, Py-H), 5.77 (d, $J=5.9 \mathrm{~Hz}, 1 \mathrm{H}, \mathrm{OH}), 5.54$ [d, $J=5.9$ $\mathrm{Hz}, 1 \mathrm{H}, \mathrm{CH}(\mathrm{OH})], 3.72\left(\mathrm{~s}, 6 \mathrm{H}, \mathrm{OCH}_{3}\right), 3.26(\mathrm{~s}, 3 \mathrm{H}$, $\left.\mathrm{NCH}_{3}\right) ;{ }^{13} \mathrm{C} \mathrm{NMR}\left(\mathrm{CDCl}_{3}, 101 \mathrm{MHz}\right) \delta: 171.44,169.63$, $166.68,153.30,133.73,131.86,130.95,129.87,128.67$, 128.61, 128.17, 123.28, 111.85, 87.97, 70.54, 54.18, 34.68. HRMS calcd for $\mathrm{C}_{21} \mathrm{H}_{20} \mathrm{Cl}_{2} \mathrm{~N}_{3} \mathrm{O}_{5}[\mathrm{M}+\mathrm{H}]^{+}$464.0775, found 464.0788 .

2,3-二氯- $N$ - $\{2-[(4,6-二 甲$ 氧基嘧啶-2-基)(羟基)甲 基]苯氧基\}- $N$-甲基苯甲酰胺(5AHb): oil, HPLC: 99.3\%; ${ }^{1} \mathrm{H}$ NMR (DMSO- $\left.d_{6}, 300 \mathrm{MHz}\right) \delta: 7.61 \sim 7.43(\mathrm{~m}, 2 \mathrm{H}$, $\mathrm{Ph}-\mathrm{H}), 7.35 \sim 7.22(\mathrm{~m}, 2 \mathrm{H}, \mathrm{Ph}-\mathrm{H}), 7.20 \sim 7.14(\mathrm{~m}, 2 \mathrm{H}$, Ph-H), $7.12 \sim 7.05$ (m, 1H, Ph-H), 6.02 (s, 1H, Py-H), 5.78 $(\mathrm{s}, 1 \mathrm{H}, \mathrm{OH}), 5.53[\mathrm{~s}, 1 \mathrm{H}, \mathrm{CH}(\mathrm{OH})], 3.73\left(\mathrm{~s}, 6 \mathrm{H}, \mathrm{OCH}_{3}\right)$, $3.23\left(\mathrm{~s}, 3 \mathrm{H}, \mathrm{NCH}_{3}\right) ;{ }^{13} \mathrm{C} \mathrm{NMR}\left(\mathrm{CDCl}_{3}, 101 \mathrm{MHz}\right) \delta$ : $171.19,168.70,154.49,136.27,133.30,131.16,130.15$, $129.46,129.11,127.11,125.20,123.43,120.48,118.24$, $111.99,88.08,71.17,54.10,34.21$. HRMS calcd for $\mathrm{C}_{21} \mathrm{H}_{19} \mathrm{Cl}_{2} \mathrm{~N}_{3} \mathrm{NaO}_{5}[\mathrm{M}+\mathrm{Na}]^{+}$486.0594, found 486.0596 .

辅助材料(Supporting Information) 所有化合物的 ${ }^{1} \mathrm{H}$ $\mathrm{NMR}$ 及 ${ }^{13} \mathrm{C}$ NMR 谱图. 这些材料可以免费从本刊网站 
(http://sioc-journal.cn/)上下载.

\section{References}

[1] (a) Lamberth, C. Heterocycles 2006, 68, 561.

(b) Ren, W.-J.; Wang, L.-Z.; Li, Y. Agrochemicals 2013, 52, 1 (in Chinese).

(任玮静, 王立增, 李云, 农药, 2013, 52, 1.)

(c) Liu, W.; Zou, X.-M.; Wang, Y.-F.; Hu, F.-Z.; Wan, Y.-Q.; Yang, H.-Z. Chem. J. Chin. Univ. 2000, 21, 237 (in Chinese).

(刘卫敏, 邹小毛, 汪义丰, 胡方中, 朱有全, 杨华铮, 高等学校 化学学报, 2000, 21, 237.)

(d) Yang, Z.-J.; Yin, C.; Zhang, L.-P.; Zhong, Y.; Xu, H.; Mao, Z.-P. Chin. J. Org. Chem.2013, 33, 2607 (in Chinese).

(杨梓君, 尹冲, 张琳萍, 钟毅, 徐红, 毛志平, 有机化学, 2013, 33, 2607.)

(e) Deng, L.-Q.; Zhong, H.; Wang, S. Chin. J. Org. Chem. 2014, 34, 414 (in Chinese).

(邓兰青, 钟宏, 王帅, 有机化学, 2014, 34, 414.)

(f) Lin, X.-F.; Tan, Z.; Liu, Y.; He, J.-P.; Bao, X.-P. Chin. J. Org. Chem. 2013, 33, 353 (in Chinese).

(林选福, 谭赞, 刘勇, 贺银菊, 鲍小平, 有机化学, 2013, 33, 353.)

(g) Bao, X.-P.; Lin, X.-F.; Jian, J.-Y.; Zhang, F.; Zou, L.-B. Chin. J. Org. Chem. 2013, 33, 995 (in Chinese).

(鲍小平, 林选福, 寒军友, 张峰, 邹林波, 有机化学, 2013, 33, 995.)

[2] (a) Feng, X.; Yao, J.-H.; Lu, L.; Tanq, Q. H.; Fan, B.-T. Acta Chim. Sinica 2006, 64, 1097.

(b) Tai, W.-J.; Chen, J.; Xu X.-Y.; Liu, Y.-J.; Xu, Y.-H.; Tang, Q.-H., Chin. J. Pestic. Sci. 2009, 11, 87 (in Chinese).

(台文俊, 陈杰, 徐小燕, 刘燕君, 许勇华, 唐庆红, 农药学学报, 2009, 11, 87.)

(c) Xu, Y.-H.; Lue, Q; Tai, W.-J.; Tang, Q.-H., Chen, J. Chin. J. Pestic. Sci. 2009, 11, 279 (in Chinese).

(许勇华, 吕强, 台文俊, 唐庆红, 陈杰, 农药学学报, 2009, 11,
279.)

[3] (a) Yoshimura, T.; Ikeuchi, T.; Ohno, S.; Asakura, S.; Hamada, Y. J. Pestic. Sci. 2013, 38, 171.

(b) Yoshimura, T.; Nakatani, M.; Asakura, S.; Hanai, R.; Hiraoka, M.; Kuwahara, S. J. Pestic. Sci. 2011, 36, 212.

[4] Lu, L.; Lu, Q.; Tang, Q.-H.; Dai, M.; Yang, B.; Xu, K.; Fu, Q.-M.; Lu, X.-T. CN 101381347, 2009 [Chem. Abstr. 2009, 310916].

[5] (a) Kawazoe, K. WO 2011024429, 2011 [Chem. Abstr. 2011, 261861].

(b) Donetti, A.; Boniardi, O.; Ezhaya, A. Synthesis 1980, 1009.

(c) Kulp, S. S.; McGee, M. J. J. Org. Chem. 1983, 48, 4097.

(d) Sauter, F.; Stanetty, P.; Mesbah, A. J. Chem. Res., Synop. 1977, 186.

[6] (a) Curtze, J.; Morschhaeuser, G.; Stumm, K.; Albert, G.; Reichert, G.; Simon, W.; Waldeck, A.; Van Tuyl Cotter, H.; Rehnig, A. E. E.; Stumm, K. O.; Cotter, H. V. T.; Morschlaeuser, G.; Rehning, A. E. E.; Stumm, A. K.; Van Tuyl, C. H.; Edi, A.; Kertz, Y.; Moschhouizer, G.; Schtum, K.; Werner, S.; Ketz, J.; Moshhoyizel, G.; Schtum, K. O.; Curtze, J.; Morschhaeuser, G.; Stumm, K.; Albert, G.; Reichert, G.; Simon, W.; Waldeck, A.; Van Tuyl Cotter, H.; Rehnig, A. E. E.; Stumm, K. O.; Cotter, H. V. T.; Morschlaeuser, G.; Rehning, A. E. E.; Stumm, A. K.; Van Tuyl, C. H.; Edi, A.; Kertz, Y.; Moschhouizer, G.; Schtum, K.; Werner, S.; Ketz, J.; Moshhoyizel, G.; Schtum, K. O. EP 897904, 1999 [Chem. Abstr. 1999, 139752].

(b) Kameswaran, V. WO 2001051440, 2001 [Chem. Abstr. 2001, 526037].

(c) Maywald, V.; Hoffmann, N.; Keil, M.; Vogelbacher, U. J.; Wevers, J. H. WO 2004054953, 2004 [Chem. Abstr. 2004, 534158].

[7] (a) Coates, R. M.; Firsan, S. J. J. Org. Chem. 1986, 51, 5198.

(b) Kozlov, M. V.; Kleymenova, A. A.; Romanova, L. I.; Konduktorov, K. A.; Smirnova, O. A.; Prasolov, V. S.; Kochetkov, S. N. Bioorg. Med. Chem. Lett. 2013, 23, 5936.

(c) Punta, C.; Rector, C. L.; Porter, N. A. Chem. Res. Toxicol. 2005, 18,349 . 